OPEN ACCESS

Edited by:

Plamen Ch. Ivanov,

Boston University, United States

Reviewed by:

Eberhard Weihe,

University of Marburg, Germany

Vincenzo Provitera,

Istituti Clinici Scientifici Maugeri (ICS

Maugeri), Italy

Sonya Bahar

University of Missouri System,

United States

${ }^{*}$ Correspondence: Peter A. Tass

ptass@stanford.edu

Specialty section:

This article was submitted to

Fractal and Network Physiology,

a section of the journal

Frontiers in Physiology

Received: 11 November 2020

Accepted: 05 February 2021

Published: 06 April 2021

Citation:

Pfeifer KJ, Kromer JA, Cook AJ, Hornbeck T, Lim EA, Mortimer BJP,

Fogarty AS, Han SS, Dhall $R$,

Halpern $C H$ and Tass PA (2021)

Coordinated Reset Vibrotactile

Stimulation Induces Sustained

Cumulative Benefits in Parkinson's

Disease. Front. Physiol. 12:624317.

doi: 10.3389/fphys.2021.624317

\section{Coordinated Reset Vibrotactile Stimulation Induces Sustained Cumulative Benefits in Parkinson's Disease}

Kristina J. Pfeifer ${ }^{1}$, Justus A. Kromer ${ }^{1}$, Alexander J. Cook ${ }^{1}$, Traci Hornbeck', Erika A. Lim ${ }^{1}$, Bruce J. P. Mortimer2, Adam S. Fogarty ${ }^{3}$, Summer S. Han ${ }^{1,4}$, Rohit Dhall ${ }^{5}$, Casey H. Halpern ${ }^{1}$ and Peter A. Tass ${ }^{1 *}$

${ }^{1}$ Department of Neurosurgery, Stanford University School of Medicine, Stanford, CA, United States, ${ }^{2}$ Engineering Acoustics, Inc., Casselberry, FL, United States, ${ }^{3}$ Department of Neurology, Stanford University School of Medicine, Stanford, CA, United States, ${ }^{4}$ Quantitative Sciences Unit, Stanford University School of Medicine, Stanford, CA, United States, ${ }^{5}$ Center for Neurodegenerative Disorders, Department of Neurology, University of Arkansas for Medical Sciences, Little Rock, AR, United States

Background: Abnormal synchronization of neuronal activity in dopaminergic circuits is related to motor impairment in Parkinson's disease (PD). Vibrotactile coordinated reset (vCR) fingertip stimulation aims to counteract excessive synchronization and induce sustained unlearning of pathologic synaptic connectivity and neuronal synchrony. Here, we report two clinical feasibility studies that examine the effect of regular and noisy vCR stimulation on PD motor symptoms. Additionally, in one clinical study (study 1), we examine cortical beta band power changes in the sensorimotor cortex. Lastly, we compare these clinical results in relation to our computational findings.

Methods: Study 1 examines six PD patients receiving noisy vCR stimulation and their cortical beta power changes after 3 months of daily therapy. Motor evaluations and at-rest electroencephalographic (EEG) recordings were assessed off medication preand post-noisy vCR. Study 2 follows three patients for $6+$ months, two of whom received daily regular $\mathrm{VCR}$ and one patient from study 1 who received daily noisy $\mathrm{VCR}$. Motor evaluations were taken at baseline, and follow-up visits were done approximately every 3 months. Computationally, in a network of leaky integrate-and-fire (LIF) neurons with spike timing-dependent plasticity, we study the differences between regular and noisy vCR by using a stimulus model that reproduces experimentally observed central neuronal phase locking.

Results: Clinically, in both studies, we observed significantly improved motor ability. EEG recordings observed from study 1 indicated a significant decrease in off-medication cortical sensorimotor high beta power $(21-30 \mathrm{~Hz})$ at rest after 3 months of daily noisy vCR therapy. Computationally, vCR and noisy vCR cause comparable parameter-robust long-lasting synaptic decoupling and neuronal desynchronization. 
Conclusion: In these feasibility studies of eight PD patients, regular vCR and noisy vCR were well tolerated, produced no side effects, and delivered sustained cumulative improvement of motor performance, which is congruent with our computational findings. In study 1, reduction of high beta band power over the sensorimotor cortex may suggest noisy vCR is effectively modulating the beta band at the cortical level, which may play a role in improved motor ability. These encouraging therapeutic results enable us to properly plan a proof-of-concept study.

Keywords: coordinated reset, vibrotactile stimulation, Parkinson's disease, desynchronization, cumulative effects, beta band power, sensorimotor

\section{INTRODUCTION}

While the hallmark of idiopathic Parkinson's disease (PD) is motor impairment, research suggests the underlying mechanism for this impairment is caused by abnormal neuronal synchrony within dopaminergic brain circuits (Hammond et al., 2007). Coordinated reset (CR) stimulation aims at long-lasting desynchronization by remodeling synaptic connectivity using specific spatiotemporal stimulus patterns (Tass and Majtanik, 2006). Computationally, it was shown that CR-induced desynchronization may reduce plastic synaptic weights, in this way causing long-lasting desynchronization and ultimately moving neural networks from stable synchronized and strong synaptically coupled states to stable desynchronized and weakly coupled states (Tass and Majtanik, 2006; Kromer and Tass, 2020). To this end, CR employs spike timing-dependent plasticity (STDP), a basic learning mechanism that modifies the strength of synapses according to the relative timing of their corresponding neurons' presynaptic and postsynaptic spikes or bursts (Markram et al., 1997; Bi and Poo, 1998; Abbott and Nelson, 2000; Caporale and Dan, 2008). CR deep brain stimulation (CR-DBS) delivered to the subthalamic nucleus (STN) for $2 \mathrm{~h}$ per day during 5 consecutive days induced acute as well as sustained motor improvement lasting for several weeks in parkinsonian monkeys (Tass et al., 2012; Wang et al., 2016). CR-DBS administered to the STN in six PD patients for $4 \mathrm{~h}$ per day for 3 days caused a significant and cumulative reduction of STN beta band activity and a correlated significant improvement of motor function (Adamchic et al., 2014).

In this study, we administer a novel noninvasive $\mathrm{CR}$ technique called vibrotactile $\mathrm{CR}(\mathrm{vCR})$ fingertip stimulation (Tass, 2017) and explore its effects on motor ability and cortical activity in PD patients. Synchronization of cortical activity is quantified by means of electroencephalographic (EEG) power (Ebersole and Milton, 2003). Following the somatosensory pathway, vibrotactile stimulation results in enhanced neuronal activity of somatic sensory ventral caudal (Vc) thalamic relay neurons (Weiss et al., 2009), as well as in the primary somatosensory cortex (SI), as shown in macaque monkeys (Harvey et al., 2013). Furthermore, neuronal activity is phase locked to skin indentation oscillations (Weiss et al., 2009; Harvey et al., 2013), which allows one to modify collective neuronal discharge patterns by controlling the timing of discharges of stimulated subpopulations (Tass, 1999). To which extent this activity propagates to other cortical areas or the basal ganglia region is still not completely understood. Stimulationrelated activity in the SI may propagate to motor areas via corticocortical connections, which has been observed in mammals across species (Jones and Powell, 1968; Jones and Powell, 1969; Mao et al., 2011). Furthermore, stimulation of the SI has been shown to elicit responses of motor cortical neurons (Kaneko et al., 1994). It is also well documented that the STN receives excitatory input from the cortical sensorimotor region via the hyperdirect pathway (Hartmannvon Monakow et al., 1978; Nambu et al., 1996), as well as from the thalamic intralaminar subnuclei CM/Pf (Sugimoto et al., 1983; Kita et al., 2016). It has been shown that neuronal activity may propagate among thalamic nuclei (Crabtree and Isaac, 2002; Crabtree, 2018). Furthermore, vibratory input may feed into motor basal ganglia thalamocortical circuits through different projections, e.g., through thalamo(Vc)-cortico(SI)striatal projections. For instance, in squirrel monkeys, it was shown by electrophysiological recordings as well as tracer injection and histology that the SI feeds into the basal ganglia by projecting to the striatum (Flaherty and Graybiel, 1991).

Of particular interest is the impact of vibratory stimulation on cortical rhythms. For instance, muscle vibration can cause a reduction in motor threshold, as measured by motor-evoked potentials derived from transcranial magnetic stimulation (Rosenkranz and Rothwell, 2003). Additionally, when participants are at rest, vibration applied to the wrist induces cortical alpha and beta power suppression over the sensorimotor cortex (Seo et al., 2019). Reduced cortical beta power over the sensorimotor cortex during motor preparation, execution, and motor imagery suggests activation of this area (Neuper et al., 1999; Neuper and Pfurtscheller, 2001; Neuper et al., 2006). These findings indicate that vibratory stimulation alone can cause activation of the sensorimotor cortex through activation of afferent pathways via vibratory stimulation.

Other vibratory stimulation techniques include whole-body vibration (WBV). WBV has been found to improve performance during upper body exercise (Marín et al., 2013) and to reduce motor symptoms in PD patients (Haas et al., 2006; Ebersbach et al., 2008; King et al., 2009). However, a recent meta-analysis suggests that the symptom-reducing effects are inconsistent (Dincher et al., 2019) and do not improve certain components of gait or balance (Lau et al., 2011). Studies examining the cortical activations involved, especially within the sensorimotor 
cortex, during and after vibration therapy in PD have not been thoroughly investigated. It may be that WBV does not have clear therapeutic benefits because it lacks effective application parameters that target specific pathological brain regions, while CR effectively reduces PD symptoms both behaviorally (Tass et al., 2012; Syrkin-Nikolau et al., 2018) and on the neuronal level by targeting specific subpopulations (Adamchic et al., 2014).

In a first-in-human study, five idiopathic PD patients received vCR fingertip stimulation for $4 \mathrm{~h}$ per day on 3 consecutive days (Syrkin-Nikolau et al., 2018). Kinematic assessments revealed improved gait and bradykinesia during stimulation and after 1 month poststimulation. However, blinded video Unified Parkinson's Disease Rating Scale (UPDRS) III scores (excluding items for rigidity and speech due to video constraints) did not show a significant change. As known from CR-DBS, a third of the pulse amplitude used for conventional high-frequency DBS caused significantly greater therapeutic effects (Tass et al., 2012), as CR requires separate stimulation of neuronal subpopulations (Tass, 2003; Tass and Majtanik, 2006). Accordingly, in the present studies, we used smaller vibration peak amplitudes (Adamchic et al., 2014) $(0.06-0.10 \mathrm{~mm})$ rather than the higher vibrational amplitude used in the first-in-human vCR study $(0.35 \mathrm{~mm}$; Syrkin-Nikolau et al., 2018).

Furthermore, we consider a randomized noisy vCR pattern. This is motivated by a previous computational study, wherein a network of leaky integrate-and-fire (LIF) neurons with STDP and electrical model stimuli, random reset (RR) stimulation was studied (Kromer and Tass, 2020). RR stimulation, achieved by adding spatial and temporal noise to the delivery mechanism of CR stimulation, may increase the robustness of long-term desynchronizing effects with respect to detuning the mean inter-stimulus interval relative to the dominant frequency of the abnormally synchronized neuronal target population (Kromer and Tass, 2020). We hypothesized that the robustness of vCR-induced long-lasting desynchronization might be improved by adding noise to stimulus delivery times.

In the present paper, we study the feasibility of vCR stimulation for the treatment of PD patients and report therapeutic effects required to design a rigorous proof-ofconcept study. We present results of two clinical feasibility studies exploring the effects of regular (i.e., non-noisy) and noisy $\mathrm{vCR}$ on PD motor symptoms. We apply vCR to a total of eight PD patients. Study 1 examines six PD patients using a noisy vCR stimulation pattern and their cortical beta band activity changes over 3 months of daily $\mathrm{vCR}$ use. Study 2 follows three patients for $6+$ months, two of whom received regular $\mathrm{VCR}$ and one patient from study 1 who received noisy vCR. Our aim in both studies was to understand how vCR affects PD patients by examining the cumulative effects of $\mathrm{vCR}$, side effects, tolerability, and neuronal changes.

We compare our clinical results to computer simulations using the same neuronal network model as previously used for the design of RR stimulation (Kromer and Tass, 2020). However, we introduce a novel stimulus model for vibratory stimuli. This model is motivated by experimental data on the response of thalamic and cortical neurons to vibratory stimulation (Weiss et al., 2009; Harvey et al., 2013). In contrast to previous stimulus models (Popovych and Tass, 2012), vibratory stimuli do not result in a phase reset of the neuronal oscillations. To deliver noisy vCR stimulation, we added jitter to the stimulus delivery times within ranges that are favorable based on proprioceptive physiology and psychophysics.

\section{MATERIALS AND METHODS}

\section{Stimulation Patterns}

In the present paper, we explored vCR stimulation as treatment for PD. Three different stimulation patterns were considered: regular vCR (Tass, 2017), the novel noisy vCR, and purely periodic multichannel stimulation (PPMS) (Zeitler and Tass, 2018). Patients in study 1 were stimulated using the noisy vCR pattern and patients in study 2 using either the regular vCR or the noisy vCR pattern. In our computational study, we considered both regular and noisy $\mathrm{vCR}$ and compared the results to those seen with vibratory PPMS (vPPMS). The three stimulation patterns are illustrated in Figure 1 and were implemented as follows.

Regular vCR stimulation, Figure $\mathbf{1 A}$, is characterized by the vCR period $T_{\mathrm{CR}}$, which sets the $\mathrm{CR}$ frequency $f_{\mathrm{CR}}=1 / T_{\mathrm{CR}}$ at which individual fingertips received burst stimuli. Individual fingertips received stimuli at multiples of $T_{\mathrm{CR}} / 4$ such that each fingertip received exactly one stimulus per CR period. Besides this constraint, stimuli were delivered to randomly selected fingertips. This type of CR stimulation is referred to as CR with rapidly varying sequences (CR RVS) in the literature (Zeitler and Tass, 2015). Additionally, we considered an $m: n$ ON-OFF pattern by delivering stimuli for an ON-period of three CR periods, $T_{\mathrm{CR}}$, and paused the stimulation for an OFF period of two CR periods afterward (Lysyansky et al., 2011). A representative regular vibrotactile 3:2 ON-OFF CR RVS pattern, in the following denoted as regular vCR, is shown in Figure 1A.

Throughout this study, we also considered a noisy version of the vibrotactile $3: 2$ ON-OFF coordinated reset with rapidly varying sequence (CR RVS) pattern, described in the previous paragraph. The noisy vCR pattern was obtained by applying a random jitter to each stimulus onset time $s$ (except for the very first stimulus). Each $s$ was drawn from a uniform distribution $s \in$ $\left[s_{0}-J \frac{T_{\mathrm{CR}}}{8}, s_{0}+J \frac{T_{\mathrm{CR}}}{8}\right]$, where $s_{0}$ is the original onset time, i.e., an integer multiple of $T_{\mathrm{CR}} / 4$, and $J$ is a jitter. $J=0 \%$ corresponds to regular $\mathrm{vCR}$, while $J>0$ leads to a noisy vCR pattern. In our clinical studies as well as in the computational model, we considered noisy vCR with $J=23.5 \%$, a representative vibrotactile noisy 3:2 ON-OFF CR RVS pattern, in the following denoted as noisy vCR, is shown in Figure 1B.

Finally, we compared results for regular and noisy vCR stimulation to vPPMS as presented by Zeitler and Tass (2018). The latter is illustrated in Figure 1C. In contrast to vCR stimulation, vPPMS considers simultaneous stimulation of all fingertips and does not enforce phase shifts between the spiking activity of individual neuronal 


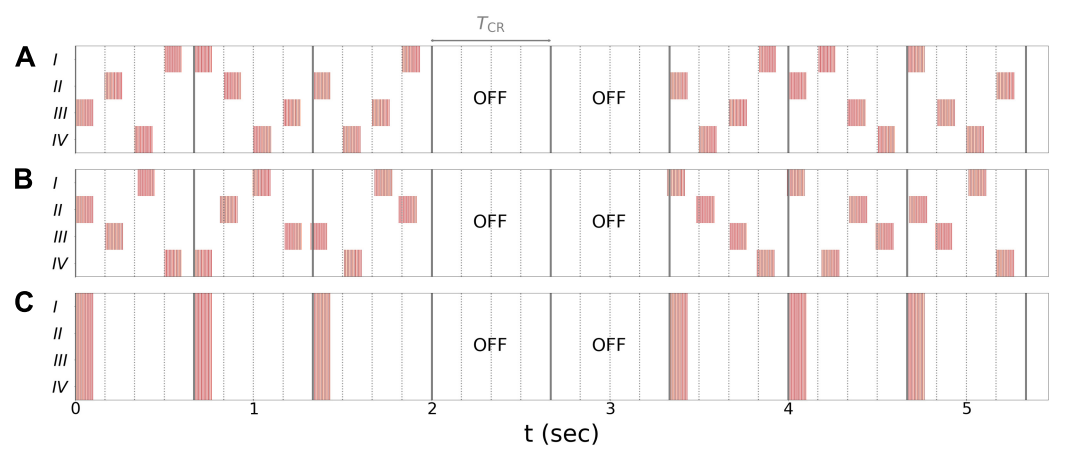

FIGURE 1 | Stimulation patterns used throughout the paper. (A) Regular 3:2 ON-OFF coordinated reset with rapidly varying sequence (CR RVS) pattern. (B) Noisy 3:2 ON-OFF CR RVS pattern and $23.5 \%$ jitter. (C) Purely periodic multichannel stimulation. Gray lines indicate multiples of the vibrotactile coordinated reset (vCR) period $T_{\mathrm{CR}}$, and dotted lines indicate multiples of $T_{\mathrm{CR}} / 4$ during individual $\mathrm{CR}$ periods. Roman numerals indicate fingertips on one hand. Stimulation bursts are marked red. Red vertical lines indicate maxima of $f_{\text {vib }}(t)$, Eq. (2), during individual bursts. Parameters: $f_{\mathrm{CR}}=1.5 \mathrm{~Hz}\left(\frac{T_{\mathrm{CR}}}{4}=166.7 \mathrm{~ms}\right)$, burst duration $100 \mathrm{~ms}$, and $f_{\text {burst }}=250 \mathrm{~Hz}$.

subpopulations (Tass, 2003; Zeitler and Tass, 2018). We therefore hypothesized poor performance with respect to long-lasting desynchronization effects.

\section{Vibrotactile Stimulation of Parkinson's Disease Patients}

For physiological design of vibrotactile glove and tactors, see Supplementary Vibrotactile Glove Material.

We used vibratory bursts of 100-ms length to achieve sufficient vibratory "loudness" (Green, 1976; Gescheider et al., 1999), while avoiding adaptation potentially caused by unnecessarily long vibratory bursts (Hahn, 1968; Goodwin, 2005; Leung et al., 2005). For noisy vCR, the jitter of stimulus times was constrained to avoid mutual masking of subsequent stimuli (Hollins et al., 1990).

For bilateral application of noisy vCR in PD patients, we used a mirrored delivery such that right and left fingers 25 were coincidently activated, respectively. This was done to avoid bilateral masking-like interference (Craig, 1985; Craig and Qian, 1997). In contrast, regular vCR was delivered to both hands in a non-mirrored manner, such that vibratory stimulus administration times were identical for both hands, but stimulus delivery was not coincident for fingers $2-5$ of both hands. This mode was chosen to increase the spatial randomization, hypothesized to be more favorable to induce long-term synaptic decoupling (Kromer and Tass, 2020).

\section{Study 1: Impact of Noisy Vibrotactile Coordinated Reset on Motor Ability and Cortical Beta Power}

\section{Participants}

Six patients were enrolled in the study after obtaining informed consent to the protocol approved by the institutional review board at Stanford University (CA, United States). All patients were clinically diagnosed with mild to moderate idiopathic PD by a staff movement disorders specialist (four men, two women, mean $(M)$ age $=53.33$ years, standard deviation
$(S D)=10.78$ years, $M$ years since $P D$ diagnosis $=9$ years, $S D=4.3$ years, Hoehn and Yahr stage $2-3, M=2.33, S D=0.51$ ). Patients were further classified as tremor-dominant $(n=4)$, postural instability/gait difficulty $(n=1)$, or intermediate $(n=1)$ (Stebbins et al., 2013). All patients were cognitively assessed using the Scales for Outcomes in Parkinson's Disease-COGnition (SCOPA-COG; Verbaan et al., 2011). No patient scored below the screening cutoff value $\leq 24$ for $\mathrm{PD}$ dementia $(M=32.16$, $S D=2.99)$. Exclusion criteria included atypical parkinsonism, presence of other neurological diseases, Hoehn and Yahr stage 1 or 5, previous brain surgery, history of skull fracture, or consumption of psychoactive medication that could alter EEG brain activity.

All patients took L-dopa or additional dopamine agonists. Prior to every visit, patients withdrew from PD medication using the following procedures: short-acting PD medication was withdrawn for $12 \mathrm{~h}$, while long-lasting [Mirapex ER (extended release), Sinemet CR (controlled release), and Requip XL (extended release)] medication was withdrawn $24-48 \mathrm{~h}$ prior to the patient's morning Movement Disorders Society-Unified Parkinson's Disease Rating Scale III evaluations (MDS-UPDRS; Goetz et al., 2008a) and EEG recordings. Levodopa equivalent daily dose (LEDD; Tomlinson et al., 2010) was calculated for each patient prior to participation according to patient reports $(M=711.50, S D=207.85)$. In addition, we collected daily medication diaries from patients online to observe medication intake. While we did not ask patients to reduce their daily medication, a 2-week average of LEDD prior to the 3-month follow-up visit was used to measure vCR effects on medication intake $(M=644.83, S D=229.85$; for patient demographics, see Supplementary Table 1).

\section{Study Procedures}

The following tests were administered on medication 1-2 weeks before vCR treatment (pretreatment screening) and 1-2 weeks before the 3-month post-vCR follow-up. These tests included the MDS-UPDRS (Goetz et al., 2008a) parts I (non-motor experiences of daily living), II (motor aspects of daily living), and IV (motor complications) and the Parkinson's Disease 
Questionnaire-39 (PDQ-39; Jenkinson et al., 1997), which measures PD-specific quality of life over the past month.

After the pre-assessment evaluations, PD patients were examined off medication over the course of 2 days using the following methods. Day 1 (baseline) consisted of a morning MDS-UPDRS III evaluation and an EEG recording (please note, on day 1 of the first visit, $\mathrm{PD}$ patients did NOT receive a full daily vCR session but did receive a short 10-min vCR stimulation period during the EEG recording). Day 2 consisted of a morning MDS-UPDRS III evaluation, followed by a vCR $2 \times 2$-h stimulation session with a 30-60-min break in between sessions. Immediately following $4 \mathrm{~h}$ of vCR stimulation, the afternoon MDS-UPDRS III evaluation occurred. Vibrotactile stimulation was delivered on digits $2-5$ to both hands (excluding the thumbs). All MDS-UPDRS III evaluations were done under the supervision of a movement disorders specialist. This set of procedures was repeated at the 3-month follow-up; however, patients continued their $2 \times 2$-h vCR session after the EEG on the first day of the 3-month follow-up. After the first initial baseline visit, patients were sent home with the custom vibrotactile device and instructed to stimulate $2 \mathrm{~h}$ in the morning and $2 \mathrm{~h}$ in the afternoon or evening on both hands until the 3-month follow-up. For a detailed schematic of the study procedures, see Figure 2.

\section{Electroencephalographic Acquisition and Recording Procedures}

High-density EEG data were collected using an EGI Net Amps 400 amplifier and a 256-electrode Hydrocel Geodesic sensor net (Magstim, Electrical Geodesics, Inc., Eugene, OR, United States). Online EEG data were digitized at 1,000 samples per second and referenced to $\mathrm{Cz}$. To ensure proper signal-to-noise ratio, impedances were kept below $50 \mathrm{k} \Omega$. EEG recordings were done in a dimly lit soundproof Faraday chamber while participants sat comfortably in a reclining chair. Participants were recorded for a total of $30 \mathrm{~min}$, which consisted of a 10-min at-rest pre-vCR baseline, a 10-min during vCR stimulation, followed by a 10 -min post-vCR recording. Patients were instructed to alternate between 1-min eyes closed and 1-min eyes open in a counterbalanced fashion throughout each of the 10min recordings.

\section{Electroencephalographic Preprocessing}

All EEG cleaning procedures were performed using Matlab R2019a and EEGlab v2019.1 (Delorme and Makeig, 2004). To minimize artifacts, only the eyes closed portions of data were used for analysis. The 1-min eyes closed epochs per condition were then chosen using the following principles. First, CR is most effective as a cumulative treatment over time (Hauptmann and Tass, 2009; Adamchic et al., 2014). Therefore, the last eyes closed epoch during vCR was used for analysis. In addition, the last 1min eyes closed epoch pre-vCR was used as a comparison. Lastly, to measure the most immediate post-vCR EEG effects, the first eyes closed epoch post-vCR was used for analysis.

The following procedures were administered to remove EEG artifacts. Data from electrodes near/on the cheeks or close to the nape of the neck were removed to reduce noise interference since these electrodes are more vulnerable to artifacts. Vertical and lateral eye electrodes were recorded from predefined areas according to the 256-electrode EGI sensor net (see Figure 3 for selected electrodes). A total of 171 electrodes including eye electrodes were used for subsequent analysis. EEG data were then average referenced and finite impulse response (FIR) filtered between 1 and $100 \mathrm{~Hz}, 6-\mathrm{dB}$ octave with a 60-Hz notch. EEG artifacts were identified and removed using the clean_rawdata EEGlab plug-in (Mullen et al., 2015). This plug-in easily identifies and separates low-frequency drifts, flatlining, and artifact-ridden channels. Parameters for electrode exclusion were set to remove a channel if flatlining for more than $5 \mathrm{~s}$, if high-frequency noise was beyond $S D=4$ of the entire channel file, and if correlations with nearby electrodes fell below 0.7. Data portions whose variance was $S D>7$ and/or $25 \%$ of electrodes went out of bounds relative to the overall data were automatically removed. In addition, artifact-ridden data identified from the clean_rawdata plug-in were visually inspected to confirm accuracy. For each EEG recording, no more than five electrodes were removed. The average length of data per 1 -min epoch was: pre-vCR $=55.42 \mathrm{~s}$, $S D=5.11 \mathrm{~s}$, during-vCR $=52.75 \mathrm{~s}, S D=7.73 \mathrm{~s}$, post-vCR $=52.54 \mathrm{~s}$, $S D=5.82$ s. After electrode and artifact rejection, data were then re-average referenced and fastICA (Hyvarinen, 1999) was run to correct for eye blinks and excessive muscle activity. In all data sets, no more than three independent component analysis (ICA) components were removed. Bad electrodes were then added back into the data set using spherical spline interpolation (Perrin et al., 1989).

\section{Source Signal Extraction}

Cleaned EEG data, with eye channels removed for source estimation, were then imported into Brainstorm (Tadel et al., 2011). The following steps were taken to create and extract source signals. The default magnetic resonance imaging (MRI) anatomy ICBM152 model was used for all subjects. Surface envelopes for the scalp, inner skull, and outer skull using the boundary element method (BEM) were generated $(1,922$ vertices per layer). MRI tissue segmentation was generated using the computational anatomy toolbox (CAT) (Gaser et al., unpublished). Here, 15,000 vertices were generated on the cortex surface, and spherical registration was used. Since our baseline EEG data were meaningful, no noise modeling was used for our noise covariance matrix. Forward modeling was then computed using OpenMEEG implemented in Brainstorm, which is based on the symmetric BEM. EEG source estimation was computed using minimum norm imaging (MNI). Standardized low-resolution brain electromagnetic tomography analysis (sLORETA; Pascual-Marqui, 2002) was used as a method to measure cortical activity, and dipole orientations were constrained to the cortex.

Power spectral density (PSD) was calculated from source activity for each frequency band of interest (Delta: $2-4 \mathrm{~Hz}$; Theta: 5-7 Hz; Alpha: 8-12 Hz; Low Beta: 13-16 Hz; Mid Beta: 17$20 \mathrm{~Hz}$; High Beta: 21-30 Hz; and Gamma: 31-50 Hz) using the Welch method with a 2 -s window overlapping by $50 \%$. Relative power (RP) was calculated by taking the sum of each frequency band and dividing it by the total power across the spectrum $(2-50 \mathrm{~Hz})$. 


\section{Study Procedures for Noisy Vibrotactile CR Treatment}

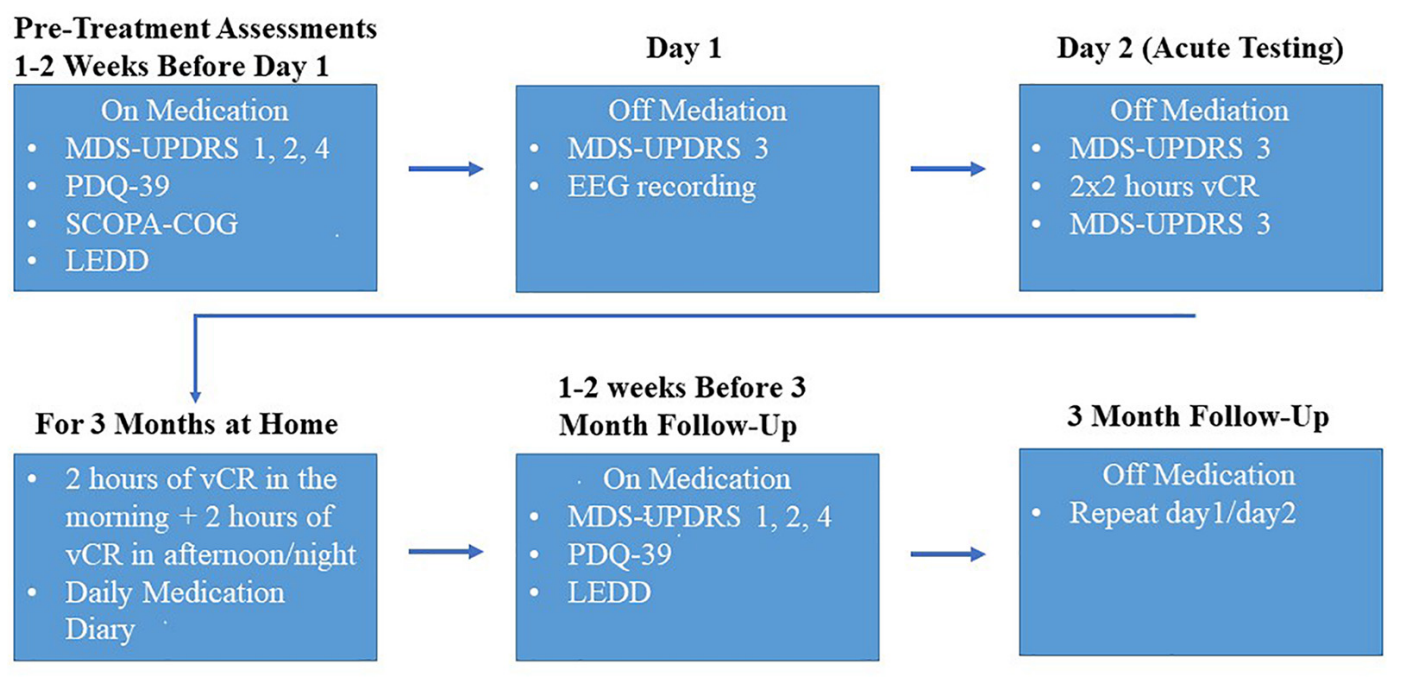

FIGURE 2 | Study procedures were as follows: 1-2 weeks before the first visit, while on medication, Parkinson's disease (PD) patients took the Movement Disorders Society-Unified Parkinson's Disease Rating Scale (MDS-UPDRS) parts 1,2, and 4, Parkinson's Disease Questionnaire-39 (PDQ-39), Scales for Outcomes in Parkinson's Disease-COGnition (SCOPA-COG) and reported levodopa equivalent daily dose (LEDD). On the first visit, PD patients were off medication and the MDS-UPDRS 3 was administered, followed by an at-rest electroencephalography (EEG). Patients withdrew their medication again overnight and on day 2 patients were assessed with a morning MDS-UPDRS part 3, followed by $2 \times 2 \mathrm{~h}$ of vibrotactile coordinated reset (VCR) stimulation and then an afternoon MDS-UPDRS part 3 (directly after $4 \mathrm{~h}$ of stimulation) to assess acute effects of vCR. After day 2, patients were instructed at home to do $2 \mathrm{~h}$ of vCR treatment in the morning and $2 \mathrm{~h}$ in the afternoon or night. The assessments described above were then repeated at 3 months of vCR treatment.

The Schaefer 200 parcellation map (Schaefer et al., 2018) was used to select a region of interest (ROI). The Schaefer parcellation map uses a gradient weighted Markov Random Field model that effectively produces homogeneous parcellations within cortical regions identified by histology or visuotopic functional MRI. The somatomotor A region (Figure 4) is broken up into 19 parcellations, all of which are considered distinct from other cortical boundaries. The somatomotor A region, which includes the hands and several other body parts but excludes auditory areas, was chosen for analysis, as it contains the most relevant regions for vibrotactile stimulation. Due to small sample size, we restricted our analysis only to the somatomotor A region rather than the whole cortex. The somatomotor A region can also be referred to the sensorimotor region, as it comprises both the primary motor and the primary somatosensory cortex. This region was chosen as the best possible area to extract sensorimotor activity, as vibration (Seo et al., 2019) and movement (Neuper et al., 1999) are known to activate the sensorimotor area.

\section{Behavioral Data Extraction and Statistical Analysis}

The MDS-UPDRS III was used as the main outcome variable for analysis. Subgroups of PD motor symptoms were subdivided based on a previous publication's classifications ( $\mathrm{Li}$ et al., 2018). The four subgroups of the MDS-UPDRS III included for analysis were: tremor (total of items 15-18), rigidity (item 3), bradykinesia (total of items 2, 4-9, and 14), and axial (total of items 1 and 9-13). Acute effects of vCR were defined as the comparison between morning MDS-UPDRS III scores (before daily stimulation) and afternoon MDS-UPDRS III (immediately after $4 \mathrm{~h}$ of stimulation) done on day 2 of the first visit and day 2 after 3 months of vCR treatment. The morning MDS-UPDRS III scores measured on day 1 of the first visit and day 1 at the 3-month follow-up visit were used to measure cumulative effects. Day 1 MDS-UPDRS III scores were chosen to analyze cumulative effects rather than day 2 scores, as patients were off medication for a prolonged amount of time on day 2. We did not want possible side effects of prolonged medication withdrawal to negatively affect our main cumulative results.

To compare mean differences between two dependent groups, a paired-samples t-test was used to analyze acute and cumulative effects. To check for clinical significance, we further compared both acute and cumulative results to minimal clinically important differences (MCID) on the MDS-UPDRS III (Horváth et al., 2015). Specifically, for acute effects, MCID scores were calculated by subtracting pretreatment (baseline) vCR MDS-UPDRS III scores from posttreatment vCR MDS-UPDRS III scores on the first visit of the second day. Cumulative MCID effects were measured by subtracting pretreatment (baseline) vCR MDSUPDRS III scores from posttreatment vCR MDS-UPDRS III scores after 3 months of vCR treatment. These difference scores will be denoted as Delta MDS-UPDRS III = post-vCR MDSUPDRS III minus pre-vCR MDS-UPDRS III. In addition, the MDS-UPDRS I, II, III, and IV, PDQ-39, and LEDD done at the pre-assessment visit and 1-2 weeks before the 3-month follow-up 


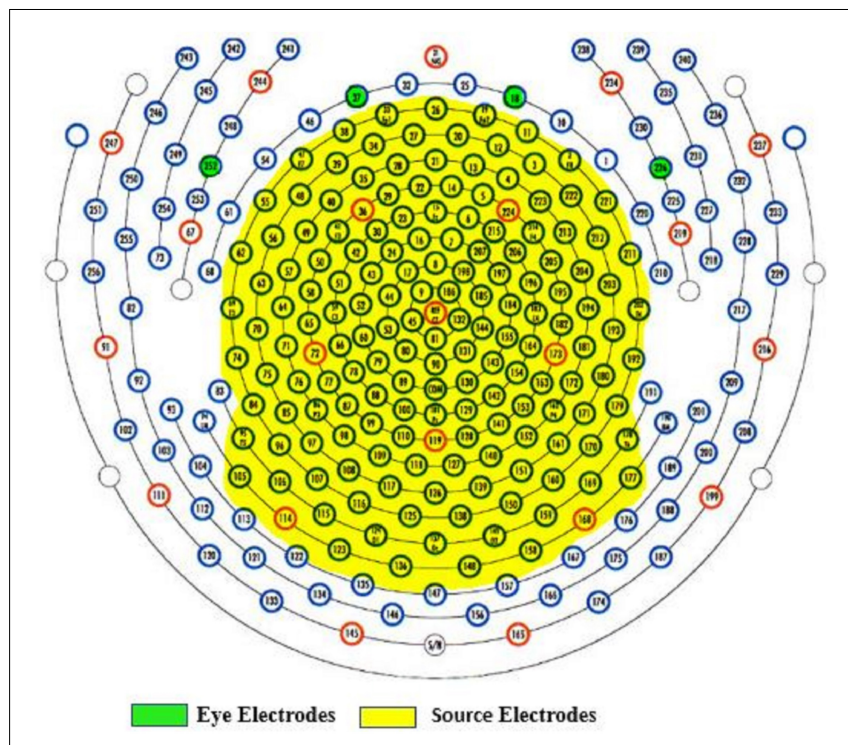

FIGURE 3 | Yellow highlighted areas indicate selected electrodes for source estimation. Data from electrodes near or on the cheeks or close to the nape of the neck were removed to reduce noise. Vertical (E18, E37) and lateral (E252, E226) eye electrodes are highlighted in green.

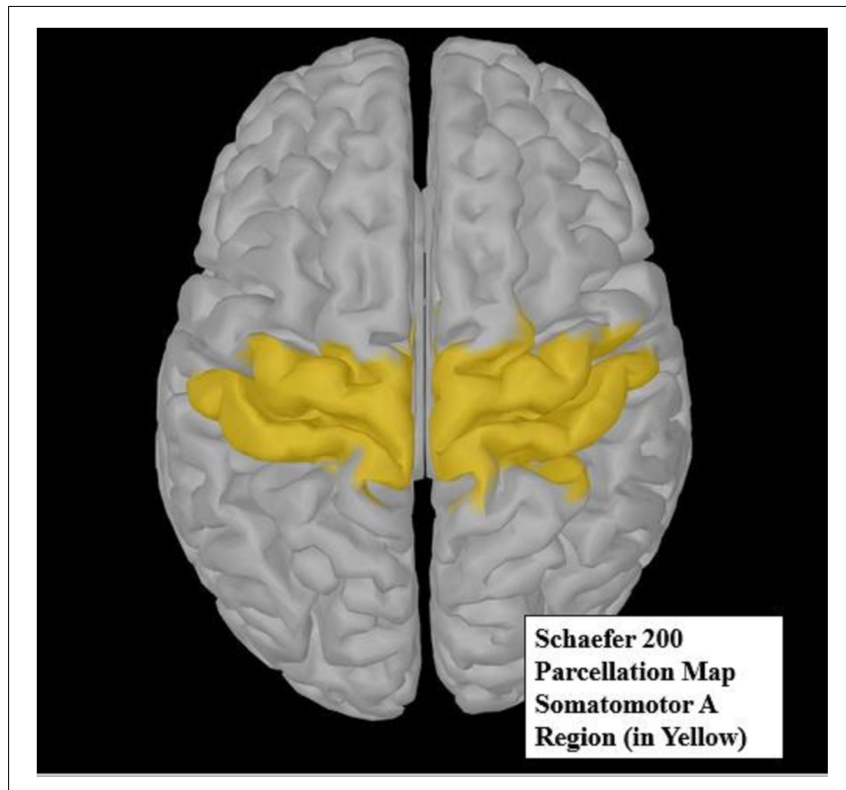

FIGURE 4 | The yellow highlighted region of the Schaefer 200 parcellation map (Schaefer et al., 2018) represents the somatomotor A region of interest (ROI). This ROI was used for source analysis.

were analyzed using a paired-samples $t$-test. Lastly, we calculated percentage decreases in LEDD, which compared baseline LEDD and 3-month LEDD. Specifically, baseline LEDD was based on patient reports at the 1-2-week pre-study assessment, and the 3-month LEDD was based on a 2 -week LEDD average collected from patient medication diaries prior to the 3month visit.

\section{Electroencephalographic Data Extraction and Statistical Analysis}

Source signals were extracted by obtaining the mean relative frequency band power of all voxels within each parcellation of the somatomotor A region. All parcellations were then averaged to obtain a single quantity of relative power at each frequency band.

Acute effects for EEG data were defined as the comparison of each 1-min eyes closed epochs (as described above), which include pre-vCR, during-vCR, and post-vCR recordings done on day 1 of the first visit and repeated on day 1 after 3 months of vCR treatment. To compare within-subject differences in relative power mean scores between pre-, during, and post-recordings of acute sensorimotor source-converted EEG data done at baseline and 3 months, separate repeatedmeasures analyses of variance (RMANOVAs) were run on each frequency band.

Cumulative effects were defined as the comparison of the 1min eyes closed pre-vCR baseline recording performed at the first study visit and the 1-min eyes closed baseline pre-vCR recordings done after 3 months of therapy. To compare relative power mean differences between two dependent groups, a paired-samples $t$ test was used to evaluate the two baseline sensorimotor sourceconverted EEG recordings.

\section{Study 2: Impact of Prolonged Vibrotactile Coordinated Reset Therapy on Parkinson's Disease Motor Symptoms}

To understand long-term cumulative effects of vCR, we followed three patients for $6+$ months. Two patients received regular vCR, while one patient from study 1 received noisy $\mathrm{vCR}$. Initially, study 1 was intended to be a 13-month trial; however, coronavirus disease 2019 (COVID19) prevented study 1 from going beyond 3 months. One patient from study 1 was able to receive MDS-UPDRS III scores remotely from his/her movement disorders neurologist. Therefore, we included this patient in study 2 and examined long-term cumulative vCR effects within all three patients.

\section{Participants}

Three patients were enrolled in the study after obtaining informed consent to the protocol approved by the institutional review board at Stanford University (CA, United States). All patients were diagnosed with idiopathic PD by a staff movement disorders specialist. For every MDS-UPDRS III rating, each patient was off PD medication for at least $12 \mathrm{~h}$. After the first initial baseline MDS-UPDRS III evaluation (pre-vCR therapy), patients received daily at-home vCR therapy. Follow-up MDSUPDRS III evaluations were done approximately every 3 months across 1-3 days.

\section{Patient 1}

Patient 1 was in his/her early 70s and was diagnosed with PD 4 years prior to participation in this study (LEDD prestudy $=450 \mathrm{mg} /$ day $)$. Patient 1 received regular vCR $(0.1 \mathrm{~mm})$ for 338 days for $2 \times 2 \mathrm{~h}$ per day, during the first 216 days to the (more affected) right hand and bilaterally thereafter. Each 
visit, the patient's motor ability was tested in the morning (before daily stimulation) and in the afternoon (directly after $4 \mathrm{~h}$ of stimulation) off medication.

\section{Patient 2}

Patient 2 was in his/her mid-50s and was diagnosed with PD 10 years prior to the start of this study. At the start of vCR, patient 2's reported LEDD was 2,700 $\mathrm{mg} / \mathrm{day}$, and he/she also took 2-3 vapes of cannabidiol/tetrahydrocannabidiol (CBD/THC). Patient 2 received bilateral regular $\mathrm{vCR}(0.1 \mathrm{~mm})$ for 185 days for $2 \mathrm{~h}$ per day. Due to the patient's reported adverse effects of medication withdrawal, this patient had off-medication assessments on the morning of the first day of each visit only.

\section{Patient 3}

Patient 3 was in his/her early 60s and diagnosed with PD 12 years prior to participation in the study. At the start of vCR therapy, patient 3's reported LEDD was $920 \mathrm{mg} /$ day. Patient 3 received bilateral noisy vCR $(0.06 \mathrm{~mm}) 2 \times 2 \mathrm{~h}$ a day for 185 days followed by a preplanned 1-month no-stimulation pause (but continuing pharmacological therapy) to assess long-lasting effects of vCR. After the preplanned 1-month follow-up, the patient continued vCR therapy but reduced his/her daily dose to a minimum of $2 \mathrm{~h}$ of stimulation three times per week for 2 months. Each visit, the patient was tested in the morning (before daily stimulation) and in the afternoon (directly after $4 \mathrm{~h}$ of stimulation) off medication.

\section{Motor Symptom Evaluations and Statistical Analysis}

The MDS-UPDRS III was used as the main outcome variable to evaluate motor symptoms by a trained movement disorders specialist. In addition, four subscores of the MDS-UPDRS III were analyzed and included: tremor, rigidity, bradykinesia, and axial, with all items selected in the same manner as in study 1. All motor scores obtained throughout the study were done off medication $(\geq 12 \mathrm{~h})$. Since each patient had a different number of vCR treatment days and number of MDS-UPDRS III scores taken, separate Pearson's $r$ correlations for each patient were used to quantify the linear relationship between morning MDS-UPDRS III scores and days of vibrotactile use. In addition, to observe acute effects for patients 1 and 3 , we report difference scores by subtracting pretreatment vCR morning scores ( $\geq 8 \mathrm{~h}$ without vCR) from posttreatment vCR motor scores (immediately after $4 \mathrm{~h}$ of vCR) done at baseline and at approximately every 3-month visit. For patient 3, we also report difference scores after the 1month pause in stimulation by subtracting 6-month MDSUPDRS III scores from 7-month MDS-UPDRS III scores. Lastly, we report baseline and posttreatment LEDD individually for all patients, and for patient 2, we report $\mathrm{CBD} / \mathrm{THC}$ amount and off times.

\section{Computational Study}

Brain regions possessing excessive neuronal synchrony during PD were modeled using a network of excitatory LIF neurons with STDP (Kromer and Tass, 2020; Kromer et al., 2020). Details on the model are given in the Supplementary Material. Parameters were chosen such that a stable synchronized and a stable desynchronized state coexisted (Kromer and
Tass, 2020; Kromer et al., 2020). Depending on their initial connectivity, networks approached either the synchronized state (strong initial connectivity) or the desynchronized state (weak initial connectivity) (see Supplementary Material for more details). Throughout the present paper, we present results on vibrotactile stimulation of networks that were prepared in the synchronized state.

\section{Vibrotactile Stimulation of the Neuronal Network Model}

We incorporated vibrotactile stimulation in our network of excitatory LIF neurons. Neurons were separated into four neuronal subpopulations, each processing afferent vibrotactile input from a single fingertip. Each subpopulation consisted of $25 \%$ of the neurons, and subpopulations did not overlap. Neurons were subject to inhomogeneous Poisson input with firing rate

$$
f_{\text {input }}(t)=f_{\text {bg }}+f_{\text {vib }}(t) .
$$

The homogeneous part, $f_{\mathrm{bg}}$, models stochastic background input from other brain regions. When a vibrotactile burst was delivered to a fingertip, related neurons were subject to additional excitatory inhomogeneous Poisson input with firing rate $f_{\mathrm{vib}}(t)$, see Eq. (1), that was present for the duration of the vibrotactile stimulation burst (duration $T=100 \mathrm{~ms}$ ). Note that transmission delays between vibratory stimulus delivery and neuronal firing rate modulation did not affect the results in the computational model as long as signals from the four fingertips experience the same delay time.

Experiments reported phase locking between skin indentation oscillations and neuronal activity of cortical neurons in primates (Harvey et al., 2013) and thalamic neurons in humans (Weiss et al., 2009). This was accounted for by a periodic modulation of the input firing rate

$$
f_{\text {vib }}(t)=A\left(1+\cos \left(2 \pi f_{\text {burst }} t\right)\right)
$$

during vibrotactile stimulation of the fingertip. $A$ is the amplitude of firing rate oscillations, and $f_{\text {burst }}$ is the frequency of vibratory oscillations against the skin. The latter were assumed to be sinusoidal, such that $f_{\mathrm{vib}}(t)$ was maximal at the highest first derivative of skin indentation oscillations (Weiss et al., 2009).

In experiments, the mean input firing rate $A$, obtained by time averaging Eq. (2) over time intervals long compared to $1 / f_{\text {burst }}$, was controlled by the amplitude of skin indentation oscillations (Harvey et al., 2013). We therefore considered it as a free parameter. We measured $A$ in units of $A_{0}$, which is the amplitude at which the total postsynaptic current was sufficient to drive the neurons' membrane potentials from reset to spiking threshold (see Supplementary Material for more details).

\section{Measure of Synchrony}

We recorded neuronal spike trains, and the degree of synchronization was quantified using the Kuramoto order parameter (Kuramoto, 1984)

$$
\rho(t)=\left|\frac{1}{N} \sum_{k=1}^{N} e^{-I \psi_{k}(t)}\right| .
$$


$\psi_{\mathrm{k}}(t)$ is the phase associated with neuron $k . \psi_{\mathrm{k}}(t)$ increased linearly between consecutive spikes by a total amount of $2 \pi$ per interspike interval (Rosenblum et al., 2001). Here, $\rho(t) \approx$ 1 and $\rho(t) \approx 0$ indicate presence and absence of in-phase synchronization, respectively.

\section{RESULTS}

\section{Study 1: The Impact of Noisy Vibrotactile Coordinated Reset on Clinical and Electroencephalographic Data}

\section{Acute Effects of Noisy Vibrotactile Coordinated Reset on the Movement Disorders Society-Unified Parkinson's Disease Rating Scale III}

In assessing the acute motor effects of noisy vCR at the outset of therapy, paired-samples $t$-tests revealed a significant effect for the MDS-UPDRS III [ $N=6, t(5)=4.297, p=0.008, S D=4.56]$. Specifically, day 2 pretreatment scores $(M=39.833, S D=11.14)$ decreased after $4 \mathrm{~h}$ of vCR stimulation $(M=31.833, S D=9.38$; Figure 5A). Axial symptom subscores showed a significant effect $[t(5)=4.719, p=0.005, S D=1.211]$, with day 2 pre-vCR scores $(M=5.33, S D=2.42)$ decreasing after $4 \mathrm{~h}$ of stimulation $(M=3.0$, $S D=2.68)$. Rigidity was also trending toward improvement $[t(5)=2.449, p=0.058, S D=2.0]$, with day 2 pretreatment scores $(M=10.166, S D=4.16)$ decreasing after $4 \mathrm{~h}$ of stimulation $(M=8.166, S D=2.9)$.

MCID for MDS-UPDRS III acute effects can be seen for each patient in Figure 6. Specifically, five out of six patients showed a clinically significant reduction of MDS-UPDRS III scores acutely on the first visit (i.e., a reduction in scores exceeding 3.25).

In assessing the acute MDS-UPDRS III effects of noisy vCR following the 3-month treatment period, a paired-samples t-test demonstrated a significant effect for rigidity subscores $[N=6, t(5)=2.907, p=0.034, S D=0.983]$. Specifically, day 2 pretreatment rigidity scores $(M=7.166, S D=3.868)$ significantly decreased after $4 \mathrm{~h}$ of vCR stimulation $(M=6.00$, $S D=3.098)$. No other significant differences were found for acute effects at 3 months.

\section{Cumulative Effects of Noisy Vibrotactile Coordinated Reset on Clinical Data}

In studying cumulative effects of noisy vCR following the 3month treatment period, a paired-samples t-test revealed a significant effect for the overall MDS-UPDRS III $[N=6$, $t(5)=2.890, p=0.034, S D=5.93]$. Specifically, day 1 pretreatment scores $(M=38.33, S D=7.86)$ significantly decreased after 3 months of vCR treatment $(32.33, S D=7.80$; Figure $5 B$ ). Subscores for tremor showed a trending effect $[t(5)=2.314$, $p=0.069, S D=5.93$ ], with day 1 pretreatment scores $(M=8.5$, $S D=4.46)$ decreasing after 3 months of vCR treatment $(M=6.66$, $S D=4.84)$. No other significant cumulative effects were found for the MDS-UPDRS I, II, and IV, PDQ-39, or LEDD pre- and post3-month treatment. LEDD was reduced on average by $7.82 \%$ after 3 months of vCR treatment.

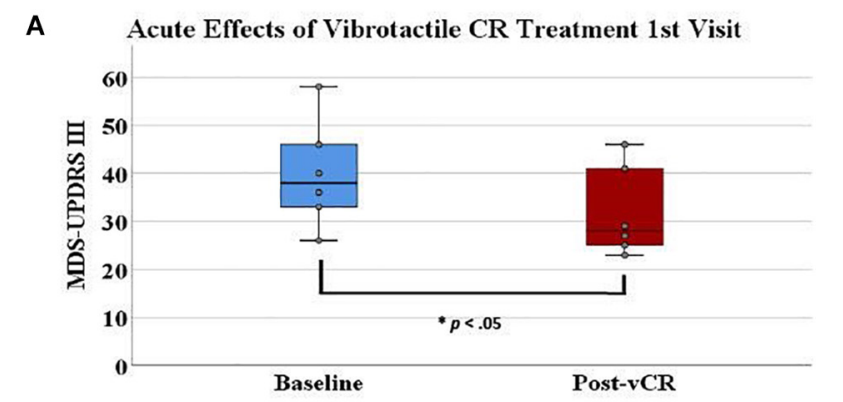

B Cumulative Effects of Noisy Vibrotactile CR Treatment

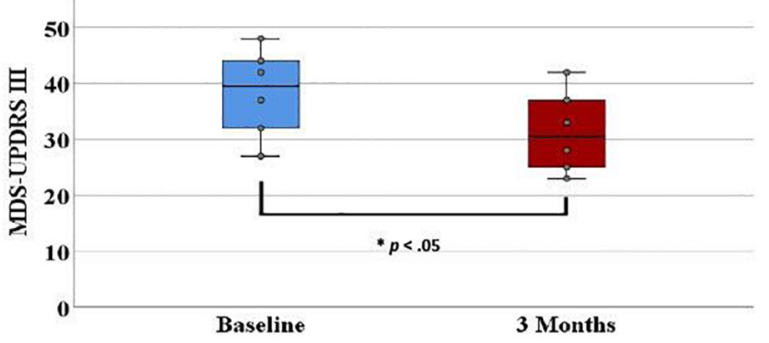

C Cummulative Effects of Regular Vibrotactile CR Treatment

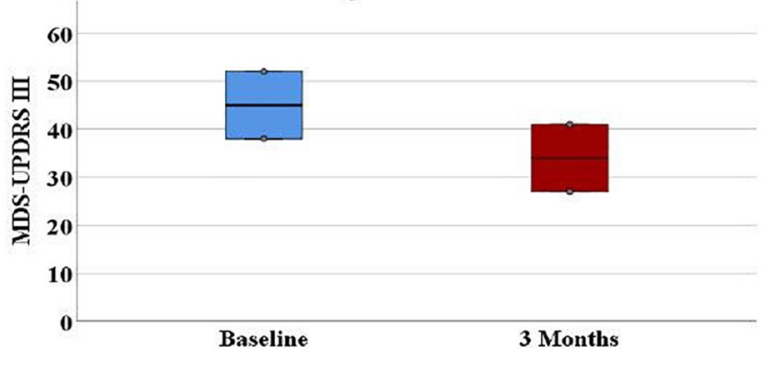

FIGURE 5 | (A) On the first visit, Parkinson's disease (PD) patients displayed a significant acute effect for vibrotactile coordinated reset (VCR) treatment. Specifically, Movement Disorders Society-Unified Parkinson's Disease Rating Scale (MDS-UPDRS) III pretreatment scores $(M=39.833, S D=11.14)$ significantly decreased after 4 h of stimulation $(M=31.833, S D=9.38)$. (B) Additionally, MDS-UPDRS III pretreatment scores $(M=38.33 \pm 7.86)$ significantly decreased after 3 months of vCR treatment $(M=32.33$, $S D=7.80)$. Panel (C) represents baseline $(M=45, S D=9.89)$ and 3-month $(M=34, S D=9.89)$ MDS-UPDRS III data for the two patients in study 2 who received regular $v C R$. While no statistics can be used due to the small sample size, regular vCR results are represented visuallyfor comparison to the noisy vCR results. Regardless of vCR type, these findings suggest significant improvement of motor ability. Panels $(\mathbf{A}, \mathbf{B})$ show box plots, whereas the boxes in panel $\mathbf{( C )}$ simply comprise the two patients' values.

Minimal clinically important differences for MDS-UPDRS III cumulative effects can be seen for each patient in Figure 6. Specifically, all six patients experienced reductions in MDSUPDRS III scores after 3 months of vCR treatment by a clinically significant amount (i.e., by more than 3.25 points; see Supplementary Video 1 for vCR effects in study 1 ).

\section{Relative Power Source Results}

One patient was unable to do the 3-month EEG recording due to COVID-19 restrictions, leaving a total of five study participants 


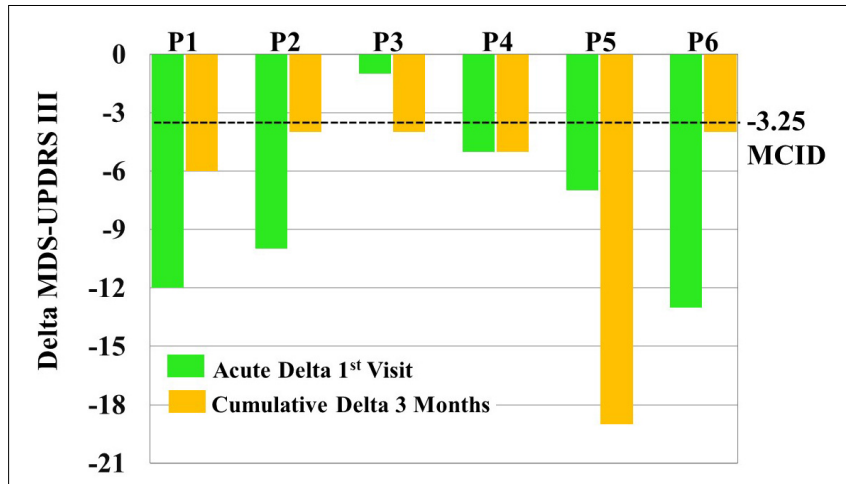

FIGURE 6 | To assess clinical significance of acute and cumulative treatment outcomes, minimal clinically important differences (MCID =-3.25) were compared to Movement Disorders Society-Unified Parkinson's Disease Rating Scale (MDS-UPDRS) score changes [i.e., Delta MDS-UPDRS

III = post-vibrotactile coordinated reset (VCR) MDS-UPDRS III minus pre-vCR MDS-UPDRS III] obtained by subtracting pretreatment (baseline) vCR MDS-UPDRS III scores from posttreatment vCR MDS-UPDRS III scores on the first visit to measure acute effects (green bars) and by subtracting pretreatment (baseline) vCR MDS-UPDRS III scores from posttreatment vCR MDS-UPDRS III scores after 3 months of vCR treatment to measure cumulative effects (orange bars). For acute effects measured on the first visit, five out of six patients were able to clinically reduce MDS-UPDRS III after $4 \mathrm{~h}$ of vCR treatment. Additionally, all patients showed a clinically significant reduction of MDS-UPDRS III scores after 3 months of vCR treatment.

included for all analyses. However, for the acute analysis done on the first study visit, we ran two separate RMANOVAs, one of which included the participant who was unable to participate in the 3-month EEG recording $(N=6)$ and one analysis in which this patient was removed $(N=5)$. In both instances, no significant acute differences in relative power for any frequency band were found between pre-vCR, during-vCR, and post-vCR EEG recordings. Additionally, at the 3-month study visit, no significant acute differences in relative power were found in any frequency band between conditions.

For cumulative effects, a paired-samples t-test demonstrated a significant effect for relative power within the high beta band $(21-30 \mathrm{~Hz})$ in the sensorimotor region $[t(4)=3.012, p=0.030$, $S D=0.015]$. Specifically, relative high beta power pre-vCR $(M=0.079, S D=0.036)$ significantly decreased after 3 months of vCR stimulation $(M=0.058, S D=0.025$; Figure 7$)$. Additionally, theta power was trending $[t(4)=-2.508, p=0.066, S D=0.0183]$, in which theta power at pre-vCR $(M=0.181, S D=0.091)$ increased after 3 months of vCR therapy $(M=0.202, S D=0.021)$.

\section{Study 2: The Impact of Prolonged Vibrotactile Coordinated Reset Therapy on Parkinson's Disease Motor Symptoms}

\section{Patient 1}

Sustained cumulative effects: Using Pearson's correlation (twotailed), we observed a significant linear decrease for the MDSUPDRS III $(N=15, r=-0.744, p=0.001)$ as well as subscores for tremor $(r=-0.712, p=0.003)$, rigidity $(r=-0.660, p=0.007)$, and bradykinesia $(r=-0.671, p=0.006)$. The axial subscore was trending ( $r=-0.492, p=0.062$; Figure 8A).

Acute effects assessed by difference scores (Delta MDSUPDRS III): Overall, patient 1 exhibited greater acute decreases in MDS-UPDRS III scores in the beginning of treatment (baseline $=-10$ ) versus the last day of treatment (post-vCR $=0$ ). For a detailed description of these difference scores, please see Supplementary Table 2 .

Medication remained at a stable pre-vCR level in between visits $(\mathrm{LEDD}=450 \mathrm{mg} /$ day). Patient 1 remained at Hohn and Yahr (HY) scale 2 on medication (pre- and with vCR).

\section{Patient 2}

Sustained cumulative effects: Patient 2 displayed a linear decrease of his/her PD motor symptoms, as Pearson's $r$ correlations demonstrated a significant decrease in total MDS-UPDRS III scores $(N=4, r=-0.998, p=0.002)$ as well as for the tremor subscores $(r=-0.978, p=0.022)$. Bradykinesia was trending in the same direction $(r=-0.940, p=0.060)$. Rigidity $(r=-0.303, p=0.697)$ and axial $(r=-0.886, p=0.114)$ were nonsignificant (Figure 8B).

Medication: From the onset of vCR, patient 2 had a reduction in medication use (LEDD decreased from 2,700 mg/day $+2-3$ vapes of CBD/THC daily to LEDD $900 \mathrm{mg} / \mathrm{day}+2-3$ vapes of CBD/THC weekly). Although we did not prospectively collect PD off time diaries, subject 2 had $>90 \%$ reduction in selfreported off time despite LEDD reduction. The latter finding, however, must be handled with caution, since it was based on self-report. Patient 2 went from HY4 on medication (pre-vCR) to HY2 on medication (with vCR), while gait improved from using a cane consistently and wheelchair occasionally to walking without assistance (see Supplementary Video 2).

\section{Patient 3}

Sustained cumulative effects: Patient 3 underwent continuous improvement of his/her motor condition, as evidenced by Pearson's $r$ correlations demonstrating a significant decrease in the MDS-UPDRS III scores $(N=5, r=-0.992, p=0.001)$ and the tremor subscores $(r=-0.976, p=0.005)$. No other significant changes were found (Figure $8 \mathrm{C}$ ).

One-month long-term effects of vCR therapy: For the MDSUPDRS III, difference scores obtained by subtracting the 6month data from 7-month data revealed minimal differences (Delta MDS-UPDRS III $=-1$ (see Supplementary Table 3 for a detailed description of difference scores of the 1-month pause in stimulation).

Acute effects assessed by difference scores: Overall, patient 3 exhibited greater acute decreases in MDS-UPDRS III scores in the beginning of treatment (baseline $=-10$ ) versus the last day of treatment at 6 months (post $\mathrm{vCR}=-1)$. For a detailed description of all difference scores from baseline to every 3-month follow-up, see Supplementary Table 3.

From the onset of vCR, patient 3 reduced medications (LEDD from $920 \mathrm{mg} /$ day at baseline to $820 \mathrm{mg} /$ day at 10 months). At the start of treatment, Patient 3 went from HY3 off medication to HY2 off medication. Patient 3 had moderate postural instability 


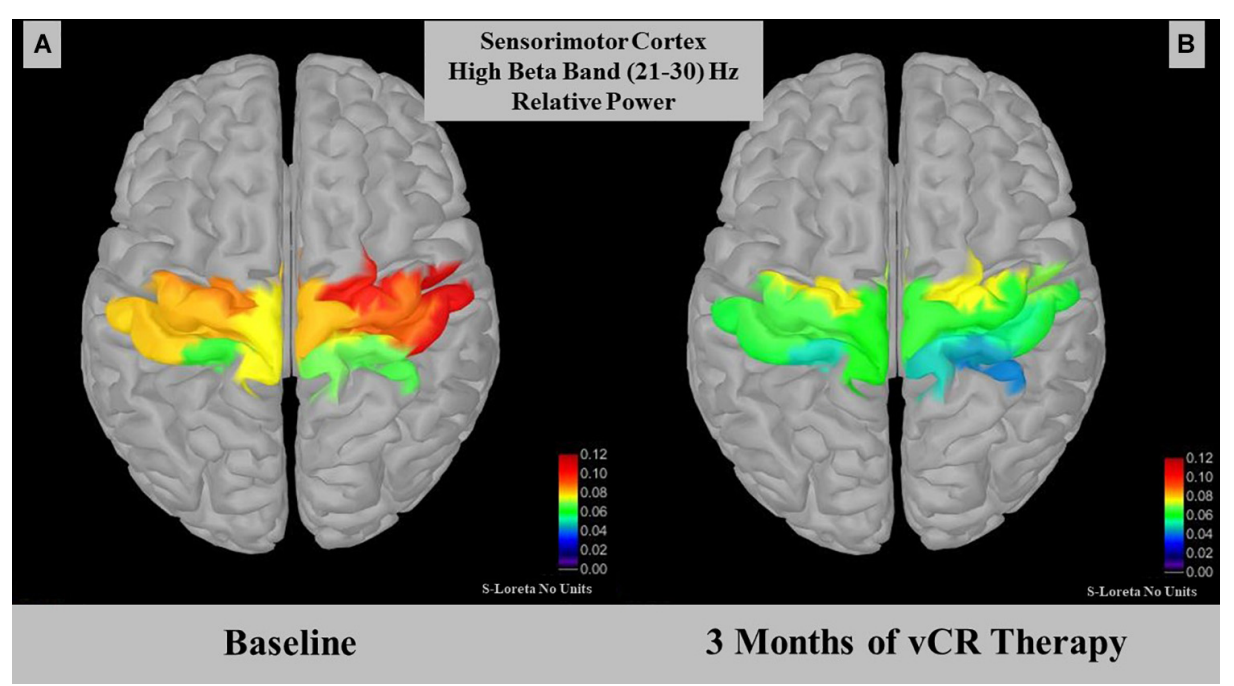

FIGURE 7 | Displays relative power for the high beta $(21-30 \mathrm{~Hz})$ band in the somatomotor A region. At-rest recordings revealed that the sensorimotor region on day 1 pre-vibrotactile coordinated reset $(\mathrm{VCR})(\mathbf{A})(M=0.079 \pm 0.036)$ significantly decreased in high beta relative power after 3 months of vCR treatment (B) $(M=0.058 \pm 0.025)$.

A

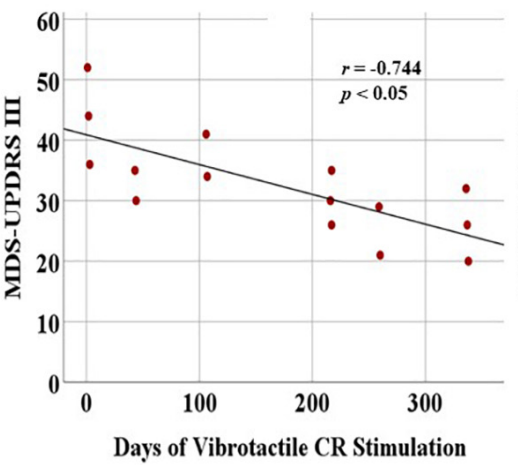

B

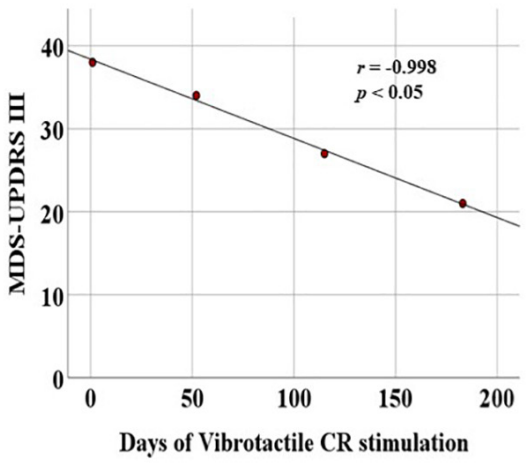

c

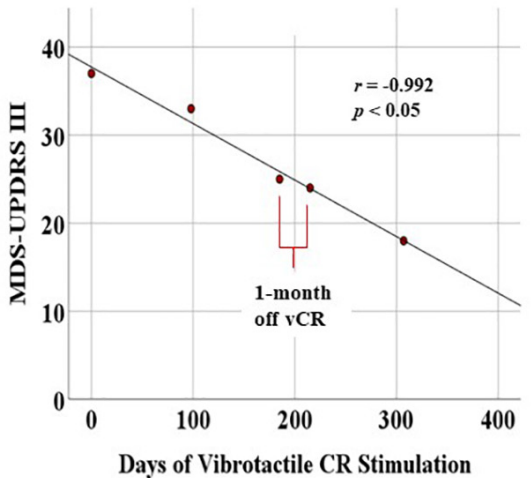

FIGURE 8 | Displays cumulative chronic, months-long effects of vibrotactile coordinated reset (vCR) treatment. For all three patients, significant negative correlations for the Movement Disorders Society-Unified Parkinson's Disease Rating Scale (MDS-UPDRS) III were found [Patient 1 (A), $r=-0.744, p=0.001 ;$ Patient 2 (B), $r=-0.998, p=0.002$; Patient 3 (C), $r=-0.992, p=0.001$ ). Patient 3 (C) also exhibited a slight decrease in MDS-UPDRS III scores at the preplanned 1-month pause in stimulation between 6 and 7 months. These results suggest significant improvement of motor ability.

at baseline to no impairment after 10 months of vCR treatment. Lastly, from months 7-10 of treatment, patient 3 was able to reduce his/her daily amount of vCR from $4 \mathrm{~h}$ to roughly $2 \mathrm{~h}$ three times per week.

On a final note, in Figure 5C, we visually compared baseline $(M=45, S D=9.89)$ and 3 -month $(M=34, S D=9.89)$ MDSUPDRS III data for the two patients in study 2 who received regular vCR to noisy vCR (Figure 5B).

\section{Computational Results}

\section{Vibrotactile Stimulation Modulates Neuronal Spiking Activity}

We studied the response of our neuronal network model in the synchronized state to vibrotactile stimulation. Raster plots of representative spiking activity during onset of stimulation are shown in Figure 9 for regular vCR (Figure 9A) and noisy vCR (Figure 9B), as well as for vPPMS (Figure 9C). We found that stimulus delivery causes collective spiking events of the stimulated neuronal subpopulation. These were followed by a complex spike pattern, resulting from spiking events caused by excitatory input from other subpopulations, especially as they received stimuli, and time periods during which neurons were irresponsive to input because their membrane potentials were far from the spiking threshold. The combination of both typically resulted in phase shifts between collective spiking events of individual subpopulations and a broadening of the distribution of spike times during these events, especially 
A
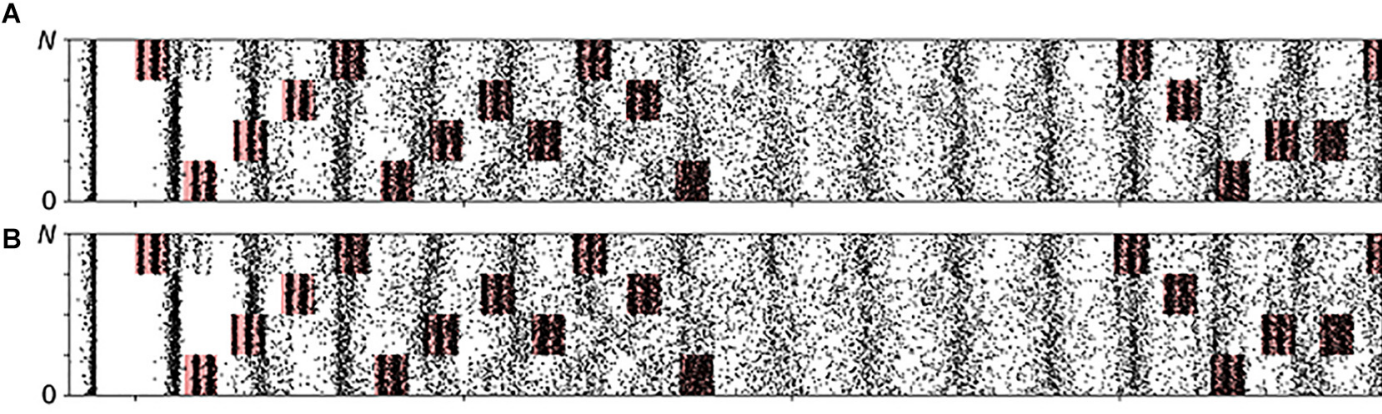

C $N$

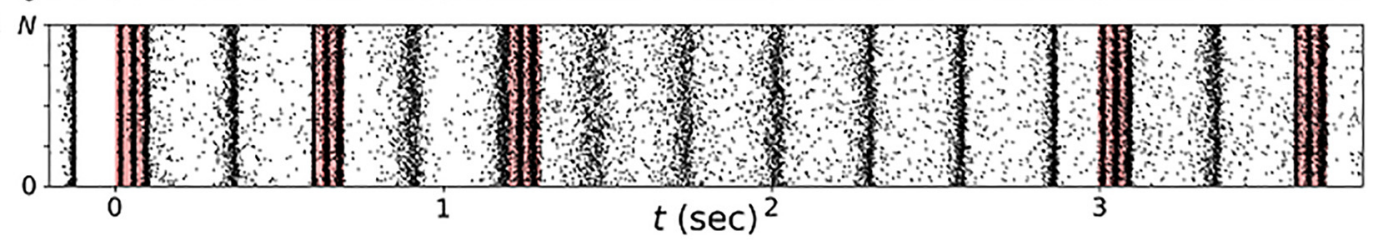

FIGURE 9 | Stimulation shapes neuronal spiking activity. Raster plots of neuronal spiking activity during the first few seconds of vibrotactile stimulation with a regular vibrotactile coordinated reset (VCR) pattern (A), a noisy vCR pattern (same sequence as regular CR but with jitter of 23.5\%) (B), and vibratory purely periodic multichannel stimulation (VPPMS) (C). Sensory inputs caused by vibratory burst delivery to corresponding fingertips are marked red. Parameters: $t=0$ marks onset of stimulation with $f_{\mathrm{CR}}=1.67 \mathrm{~Hz}$ and $A=A_{0}$.

during $\mathrm{ON}$ periods. In contrast, the collective rhythm remained intact during vPPMS, and only broadening of the distribution of spike times during collective spiking events was observed.

\section{Long-Lasting Desynchronization by Vibrotactile Coordinated Reset Stimulation}

We studied acute and long-lasting effects of noisy vCR stimulation in the neuronal network model. vCR was delivered for $1 \mathrm{~h}$ to networks in the synchronized state. To evaluate acute effects, the mean synaptic weight $\langle w\rangle(t)$ and the Kuramoto order parameter $\rho(t)$, Eq. (3), were calculated during stimulation. After cessation of stimulation, the simulation was continued to study long-lasting effects.

Representative trajectories of $\rho(t)$ and $\langle w\rangle(t)$ before, during, and after noisy vCR stimulation are shown in Figure 10. We found that $\rho(t)$ decreased during stimulation, demonstrating acute desynchronization in response to ongoing noisy $\mathrm{vCR}$ stimulation. Furthermore, $\langle w\rangle(t)$ reduced gradually and finally approached a stationary value, indicating stimulation-induced decoupling of the neurons. After cessation of stimulation, $\langle w\rangle(t)$ further decreased and the spiking remained desynchronized, indicating that stimulation drove the network into the basin of attraction of a stable desynchronized state.

In addition to the mean synaptic weight, Figure 10A, we analyzed the specific structure of the connectivity matrix in Figure 10B. As predicted in our previous work (Kromer and Tass, 2020), we found qualitative differences between the mean weight of synapses interconnecting different neuronal subpopulations and synapses connecting neurons in the same subpopulation (Figure 10B). In the following, these synapses are referred to as interpopulation and intrapopulation synapses, respectively. While interpopulation synapses weakened during stimulation, weights of intrapopulation synapses remained strong or even strengthened [Figure 10B $(1 \mathrm{~h})$ ]. Thus, in the neuronal network model, noisy vCR stimulation mainly weakened interpopulation synapses. Nevertheless, stimulation led to longlasting desynchronization (Figure 10A). Thus, weakening of interpopulation synapses was sufficient to drive the network into the basin of attraction of a stable desynchronized state.

\section{Acute and Long-Lasting Effects of Vibrotactile Stimulation}

Next, we studied the parameter dependence of acute and longlasting effects of vibrotactile stimulation in the neuronal network model. First, we considered the degree of acute synchronization as quantified by the Kuramoto order parameter, Eq. (3). Figure 11 displays results for regular vCR (A), noisy vCR (B), and vPPMS (C). We found that both vCR stimulation protocols caused pronounced acute desynchronization for sufficiently fast and strong stimulation and a wide range of stimulation amplitudes. In contrast, spiking remained synchronized during vPPMS.

In particular, we show the mean synaptic weight (second column) and the mean weights of intrapopulation (third column) and interpopulation synapses (fourth column) in Figure 11. Note that we did not distinguish between the latter two for vPPMS, as all neurons received stimuli simultaneously. We found a considerable decrease of the mean synaptic weight in the parameter regions where stimulation led to acute desynchronization. We further found that $\mathrm{CR}$ stimulation for which $T_{\mathrm{CR}} / 4$ is smaller than the period of the synchronous rhythm, $1 / f_{\text {synch }}$, caused more robust weight reduction with respect to the mean input firing rate $A$. For slower stimulation, weight reduction was limited to certain values of $A$. In contrast, vPPMS did not weaken synapses, instead it might even increase the mean synaptic weight for fast and strong stimulation, see Figure 11C. 


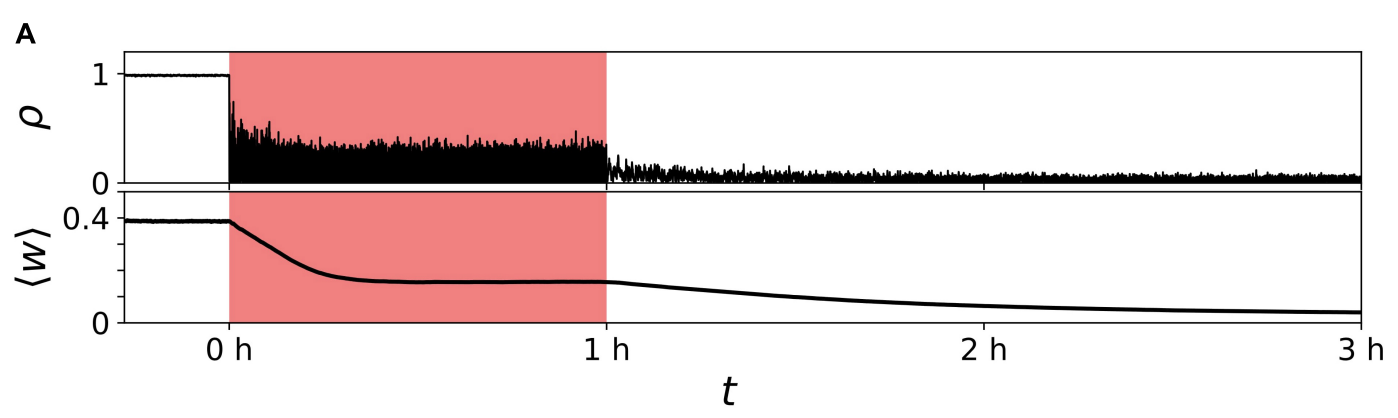

B
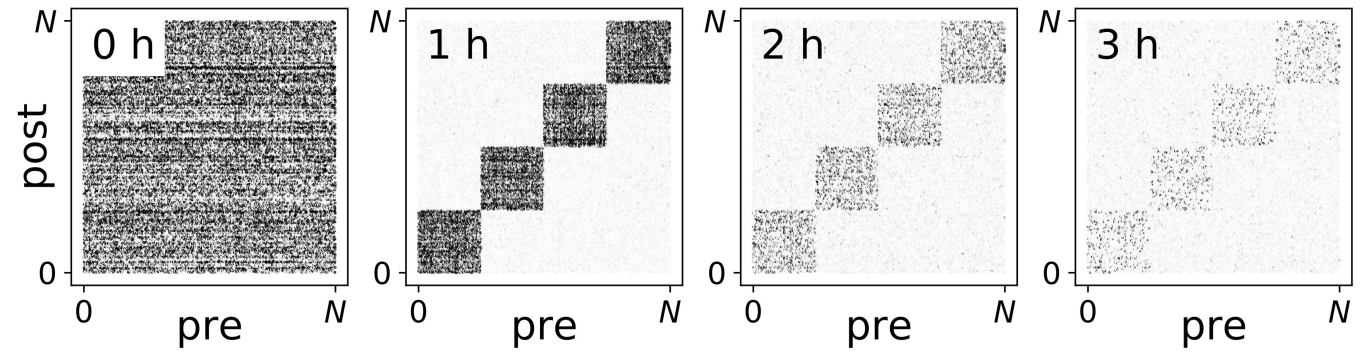

FIGURE 10 | Long-lasting desynchronization by sensory stimulation with noisy vibrotactile coordinated reset (vCR). (A) Time trace of the Kuramoto order parameter $\rho(t)$ and the mean synaptic weight $\langle W\rangle(t)$ before, during (red), and after noisy vCR stimulation. (B) Snapshots of connectivity matrices containing the values of all synaptic weights $w_{i \rightarrow j}(t)$ evaluated at indicated times after onset of stimulation; see also labels in panel (A). Parameters: $J=23.5 \%, f_{\mathrm{CR}}=1.5 \mathrm{~Hz}$, and $A=A_{0}$.

We found qualitative differences between the dynamics of intrapopulation and interpopulation synapses during vCR stimulation. Intrapopulation synapses only weakened in a small portion of the parameter space, mostly for a welldefined mean input firing rate $A$ (Figures 11A,B). In the remaining part, intrapopulation weights typically increased. Thus, vCR stimulation strengthened synaptic connections between neurons responding to the same vibrotactile bursts. In consequence, decoupling was mainly driven by a stimulation-induced reduction of interpopulation synapses. The latter occurred primarily in parameter regions where acute desynchronization was observed.

We found poor performance of $\mathrm{VCR}$ stimulation when $T_{\mathrm{CR}} / 4$ was close to the inverse frequency

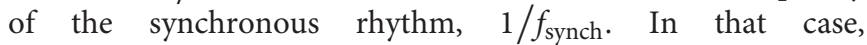
stimulation stabilized the collective rhythm rather than inducing phase shifts between rhythms of individual neuronal subpopulations.

Too slow and/or weak stimulation was not capable of shifting spiking rhythms of separately stimulated subpopulations against each other reliably and caused only weak decoupling. On the other hand, too strong stimulation caused high firing rates during stimulus delivery. Then, the stimulated subpopulation provided strong excitatory input to the others, and their spiking rhythms aligned. Furthermore, strong stimulation caused a strengthening of intrapopulation weights. For intermediate stimulation amplitudes ( $A$ of the order of $A_{0}$ ), we found a reliable reduction of the mean synaptic weight for a broad range of interstimulus intervals.
Next, we considered long-lasting effects of the different stimulation protocols. Simulation results for regular and noisy vCR stimulation are presented in Figures 11D,E. We found that regular vCR (Figure 11D) and noisy vCR (Figure 11E) showed pronounced long-lasting effects in parameter regions, where acute desynchronization was found (compare Figure $11 \mathrm{D}$ to Figure $11 \mathrm{~A}$ and Figure $11 \mathrm{E}$ to Figure 11B, respectively). In contrast, vPPMS did not entail long-lasting desynchronization or changes of the mean synaptic weight (Figure 11F).

\section{DISCUSSION}

We studied the effects of $\mathrm{vCR}$ stimulation on PD patients in two clinical feasibility studies and in a computational neuronal network model with STDP. In the following, we discuss the results of our clinical studies and compare them to the effects of vibrotactile stimulation in the computational model.

\section{Study 1: The Impact of Noisy Vibrotactile Coordinated Reset on Motor and Cortical Beta Power Changes}

Acute decreases in the MDS-UPDRS III after vCR treatment were mainly seen on day 1 . At month 3, acute decreases in the MDS-UPDRS III after vCR treatment were only significant for tremor subscores. This may suggest that acute effects reduce over time, which is expected as the brain adapts to a more normalized state. MDS-UPDRS III 

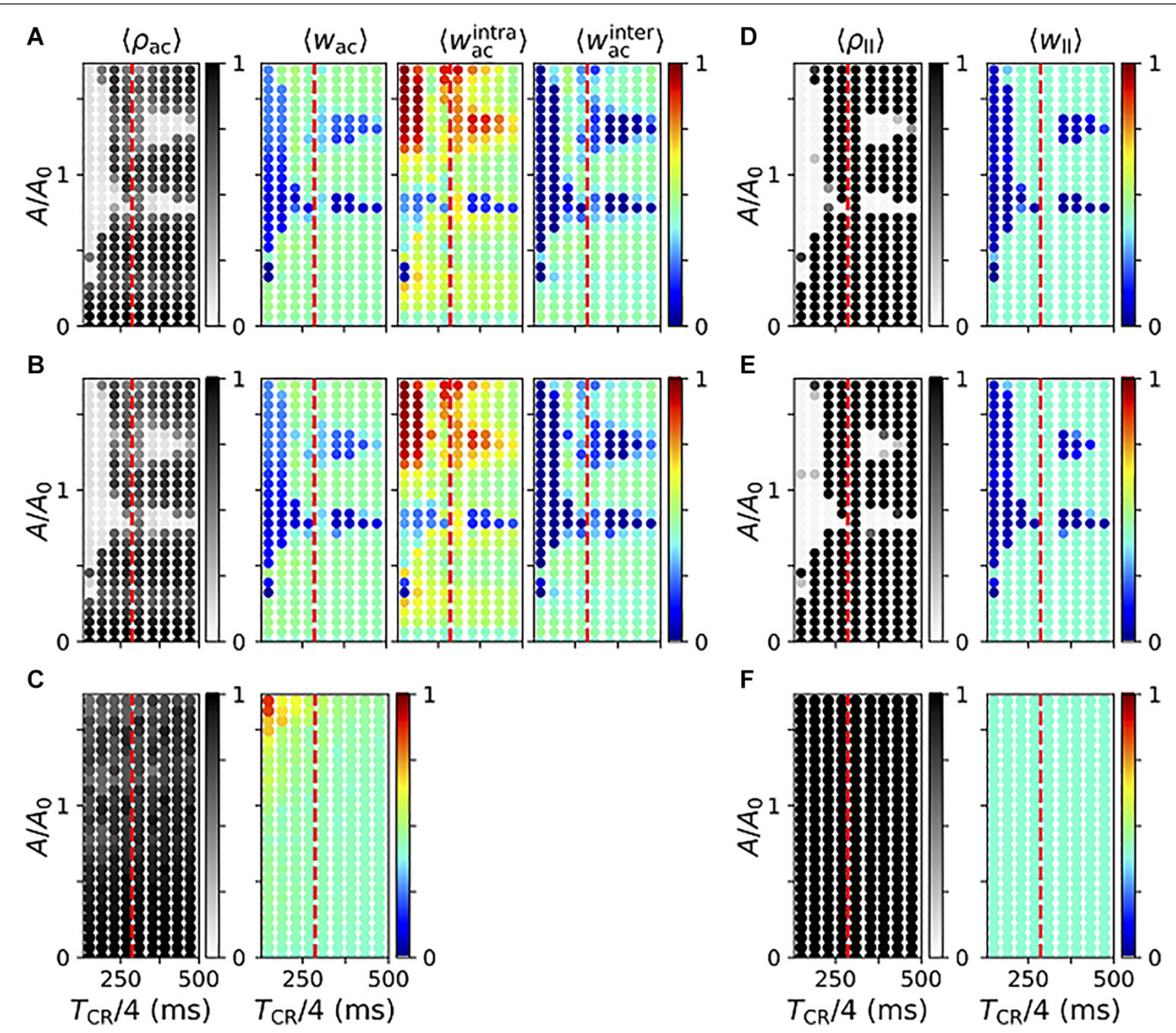

FIGURE 11 | Parameter dependence of stimulation-induced desynchronization and weight dynamics in the neuronal network model. (A-C) Acute effects of regular vibrotactile coordinated reset (vCR) (A), noisy vCR (B), and vibratory purely periodic multichannel stimulation (vPPMS) (C). Columns show results for the acute Kuramoto order parameter $\rho_{\mathrm{ac}}$ evaluated shortly before stimulation ceases (first column); the corresponding mean synaptic weight $W_{\mathrm{ac}}$ (second column); and mean weights of intrapopulation (third column) and interpopulation synapses (fourth column), respectively. (D-F) Corresponding long-lasting effects of regular vCR (D), noisy vCR (E), and vPPMS (F) evaluated $1 \mathrm{~h}$ after cessation of stimulation. Here, the first column shows the Kuramoto order parameter $\rho_{\|}$and the second column the mean synaptic weight $w_{\|}$. For comparison, the mean period of the original synchronous rhythm $\left(1 / f_{\text {synch }}, f_{\text {synch }}=286\right.$ ms $)$ is marked by vertical dashed red lines. All results were time-averaged over an interval of $12 \mathrm{~s}$ shortly before cessation of stimulation (A-C) and $1 \mathrm{~h}$ after cessation of stimulation (D-F). Data points show ensemble averages (marked by angular brackets) over five network and sequence realizations. Results for noisy vCR were obtained using a jitter of $23.5 \%$.

scores significantly decreased after 3 months of vCR treatment. This finding may suggest that vCR motor improvement effects can be long lasting. Lastly, after 3 months of vCR treatment, patients showed no significant changes in the MDS-UPDRS I, II, and IV, PDQ-39, or LEDD compared to the first study visit. However, percentage decreases indicated that LEDD decreased by $7.82 \%$ on average. This is important, as it suggests that patients may decrease their LEDD when receiving vCR treatment, which in turn may provide substantial therapeutic relief from dopaminergic side effects. A greater sample size in future studies will address this finding further.

Acute relative EEG power vCR effects were not significant on the first visit or at the 3-month follow-up visit. Considering acute effects on the first visit were seen in MDS-UPDRS III scores after $2 \mathrm{~h}$ of stimulation, $10 \mathrm{~min}$ of $\mathrm{vCR}$ stimulation during the EEG recording may be an insufficient amount of time to cause significant cortical changes. Indeed, $\mathrm{CR}$ is a cumulative treatment, in which effects become greater after a sufficient amount of time (Hauptmann and Tass, 2009; Adamchic et al., 2014).

High beta band relative power $(21-30 \mathrm{~Hz})$ significantly decreased over the sensorimotor cortex after 3 months of vCR treatment. Studies have shown that PD patients display beta band coherence between motor cortical EEG areas and the STN (Marsden et al., 2001; Williams et al., 2002). Attenuation of synchronous beta activity in the STN occurs while PD patients are under the influence of L-dopa (Brown et al., 2001; Weinberger et al., 2006; Giannicola et al., 2010) or when DBS is delivered to the STN (Bronte-Stewart et al., 2009; Whitmer et al., 2012). Previous studies have suggested that the beta band can be subdivided into low and high bands, with low beta band activity decreasing in the STN when PD patients receive dopaminergic therapy (Priori et al., 2004; López-Azcárate et al., 2010). In addition, the high beta band has been associated mainly with gait activity, with increases in power relating to impaired freezing of 
gait (FoG) in cortical EEG motor areas (Ly et al., 2016) and in the STN (Toledo et al., 2014). Low beta activity has been related to bradykinesia and rigidity in the STN (López-Azcárate et al., 2010). While our sample size is too small to make significant claims regarding subtypes of $\mathrm{PD}$, the relationship between the beta band power decrease in sensorimotor cortex after 3 months of vCR treatment is promising, as it suggests vCR therapy may modulate power in this band.

\section{Study 2: The Impact of Prolonged Vibrotactile Coordinated Reset Therapy on Parkinson's Disease Motor Symptoms}

All patients showed significant cumulative improvement in the MDS-UPDRS III. Patients 1 and 3 showed greater acute effects of vCR treatment in the beginning of the study compared to later follow-up visits. For LEDD amount, no patient increased his/her medication throughout the study. Patient 1 was able to maintain his/her medication and continuously improve motor ability. Patients 2 and 3 experienced a reduction in medication while constantly improving their motor scores. For patient 2, we also considered that patient 2 may have had "supraon state FoG" (Espay et al., 2012) with improvement in FoG after vCR related to reduction of LEDD. However, patient and spouse reported that when FoG occurred, it reliably improved by taking short-acting L-dopa, arguing against this. Taking additional doses did not result in reoccurrence of FoG, but in dyskinesia. Patient 3 showed no notable changes in MDSUPDRS III scores when comparing the 6-month data to the data at 7 months, i.e., after a preplanned 1-month pause in vCR stimulation. Additionally, this patient reduced his/her daily $4 \mathrm{~h}$ of vCR time to approximately $2 \mathrm{~h} 3$ times a week after the 7-month follow-up and still showed exceptional improvements in the MDS-UPDRS III scores, as displayed in Figure 8C.

The cumulative decrease of off-medication MDS-UPDRS III scores observed in this study is remarkable since in PD patients, MDS-UPDRS motor scores typically increase over time. For instance, in a study in 362 patients with de novo $\mathrm{PD}$, a linear increase of MDS-UPDRS scores was observed over 5 years, with an estimated 4.9 increase of total MDS-UPDRS per year (Holden et al., 2018).

Overall, in both studies, vCR was easily managed, yielded no side effects, and delivered significant cumulative and sustained improvement in the MDS-UPDRS III scores.

\section{Vibratory Displacement Effects}

The first-in-human study using $0.35-\mathrm{mm}$ vibration amplitude found no significant differences in the UPDRS III during the 3-day treatment phase (Syrkin-Nikolau et al., 2018). However, items for rigidity and speech were excluded in this study, which makes comparing our results difficult. Nevertheless, our finding of strong acute decreases in the MDS-UPDRS III observed during study 1's first visit and in study 2 in patient 1's first 3-day visit, and patient 3's first day visit may indicate that smaller peak vibration amplitudes $(0.1 \mathrm{~mm} / 0.06 \mathrm{~mm})$ are more beneficial toward treating patients' motor symptoms.
The majority of mechanoreceptors of the glabrous skin of the human hand are fast adapting (FA), where FA I mechanoreceptors respond to $30-60-\mathrm{Hz}$ vibrations, and FA II mechanoreceptors to 100-300 Hz (Johansson and Vallbo, 1983). Conduction velocities of FA I and FA II mechanoreceptors are in similar ranges (Knibestöl, 1973) but may still be different enough to compromise the vCR activation pattern. Smaller vibration amplitudes might be more beneficial for two reasons: (1) Smaller-amplitude $250-\mathrm{Hz}$ vibrations might stimulate the FA II mechanoreceptors more selectively, which might be favorable in case of larger differences in conduction velocities of FA I and FA II mechanoreceptors; for details, see Tass (2017). (2) Smaller-amplitude $250-\mathrm{Hz}$ vibrations of different fingertips might activate cortical representation areas with smaller spatial overlap, which is more favorable for CR stimulation; for details, see Tass (2017). In the computational model, we find better performance at moderate amplitudes as well. Here, high mean input firing rates corresponding to large amplitudes of skin indentation oscillations (Harvey et al., 2013) cause high firing rates of stimulated neurons during stimulus deliveries. Then, the stimulated subpopulation provides strong excitatory input to the other subpopulations that may result in synchronization of the spiking rhythms of separately stimulated subpopulations. Therefore, strong stimulation reduces phase shifts between the rhythms of individual subpopulations and reduces acute desynchronization effects.

\section{Limitations and Future Directions of Clinical Studies}

Our clinical studies demonstrate encouraging therapeutic effects of chronic, months-long vCR therapy. Nevertheless, limiting factors prevent us from making strong conclusions regarding the efficacy of $\mathrm{vCR}$ to the general PD population because of a small sample size and lack of sham condition. However, we do not believe this treatment to be the result of a placebo effect for the following reasons: (1) It has been previously shown in PD patients that increases in motor function due to a placebo effect are longitudinally nonuniform, while our patients responded in a uniform way (Goetz et al., 2000). (2) Tremor is less susceptible to the placebo effect (Goetz et al., 2000). In our studies, study 1 showed trending cumulative decreases in tremor at 3 months of vCR treatment, and study 2 showed significant cumulative decreases in tremor for all patients. (3) In medical and surgical interventions, the overall positive placebo response rate using the MDS-UPDRS III is 16\% (Goetz et al., 2008b). Our percentage decrease in MDS-UPDRS III at 3 months was $15.65 \%$. However, given that vCR treatment is most effective when used for a longer period of time (Adamchic et al., 2014; Hauptmann and Tass, 2009), our 6+-month data may be a more reliable measure. For instance, when comparing MDSUPDRS III baseline scores to the last day of treatment in study 2 , the overall percentage decrease was $53.52 \%$, over three times the expected placebo effect seen in long-term PD studies (Goetz et al., 2008b).

Study 1 was initially planned as a comparative 13-month study in $2 \times 10$ PD patients comparing the effects of regular vCR and 
noisy vCR (6 months of 2-4 h of regular vs. noisy vCR per day, followed by a preplanned 1-month vCR pause to assess whether vCR effects are long lasting, finally followed by 6 months of $2 \mathrm{~h}$ of regular vs. noisy CR three times per week). Due to COVID19 , only six patients in the noisy vCR group were able to finish the 3-month follow-up. Based on the clinical results presented here, we cannot infer outcome differences between regular vCR and noisy vCR, which is in agreement with our computational results presented.

\section{Computational Studies}

We studied long-lasting effects of vibrotactile stimulation in a network of excitatory LIF neurons with STDP. Prior to stimulation, neuronal activity was synchronized. This model network represented a brain region expressing excessive neuronal synchrony during PD. Neurons were subject to excitatory input from a brain region with increased and periodically modulated spiking activity during vibrotactile stimulation. Delivery of vibratory burst stimuli resulted in a substantial increase and a periodic modulation of the input firing rate of this brain region, as observed experimentally in the human somatosensory thalamic nucleus (Weiss et al., 2009) and SI of macaque monkeys (Harvey et al., 2013). This affected pathological synchrony and caused desynchronization. Furthermore, it triggered a plastic reorganization of the network, which led to long-lasting changes of spiking activity. Future computational studies will be devoted to refining the modeling approach by taking into account more complex and physiologically more realistic network models that incorporate collective bursting (Powanwe and Longtin, 2019) and allow for studying the effect of stimulation on multiple coexisting pathological rhythms and cross-frequency coupling (Hyafil et al., 2015).

\section{Comparison With Effects Caused by Electrical Model Stimuli}

We found qualitatively different reshaping of intrapopulation and interpopulation synapses during application of regular and noisy vCR stimulation (Figures 11A,B). While intrapopulation synapses typically strengthened during stimulation, interpopulation synapses weakened. Weakening of interpopulation synapses was in accordance with previous results on electrical multisite CR stimulation presented in Kromer et al. (2020). However, results for intrapopulation synapses differed qualitatively. In more detail, Kromer et al. (2020) reported a weakening of intrapopulation synapses during electrical CR stimulation. This discrepancy may result from different statistics of neuronal spiking responses to administered stimuli. A recent theoretical study on networks of LIF neurons with STDP (Kromer and Tass, 2020) revealed two basic mechanisms for reshaping of network connectivity in response to spatiotemporal stimulus patterns: sequence and stimulus-induced reshaping. Sequence-induced reshaping describes synaptic reshaping as a consequence of the statistics of interstimulus intervals between stimuli administered to individual neuronal subpopulations. In contrast, stimulus-induced reshaping describes synaptic reshaping as a consequence of neuronal responses to individual stimuli. During CR stimulation, the former strongly affects interpopulation synapses, whereas the latter dominates the dynamics of intrapopulation synapses (Kromer and Tass, 2020). We expect that qualitatively similar results for interpopulation synapses during vCR and electrical CR stimulation were caused by the characteristic statistics of time lags in the CR pattern, used here and in Kromer et al. (2020). In contrast, individual stimuli used here and in Kromer et al. (2020) differed significantly. In Kromer et al. (2020), short, charged-balanced electrical pulses were used. These pulses resulted in a sharp collective spiking response of stimulated neurons. This led to a weakening of intrapopulation synapses by a delay-induced effect introduced as “decoupling through synchrony" by Lubenov and Siapas (2008). In contrast to short electrical stimuli, we modeled vibrotactile stimuli by a significant increase and modulation of the firing rate of presynaptic Poisson input. The distribution of spike times during individual collective spiking responses was rather broad and extended over tens of milliseconds (see raster plots in Figure 9). As discussed in Knoblauch et al. (2012), Kromer and Tass (2020), Lubenov and Siapas (2008), distributions that were broad compared to the axonal delays $(3 \mathrm{~ms}$ in our model) led to synaptic potentiation, as presynaptic spikes often arrived before postsynaptic ones. This led to the strengthening of intrapopulation synapses in our neuronal network model (Figure 11). For a comparison with previously employed sensory model stimuli, we refer to Supplementary Discussion 3.1.

\section{Comparison to Purely Periodic Multichannel Stimulation}

We compared simulation results for regular and noisy vCR stimulation to those for vPPMS. Purely periodic multichannel stimulation (PPMS) was originally introduced in Zeitler and Tass (2018) and corresponded to the simultaneous stimulation of all four fingertips at the beginning of the CR cycles in a 3:2 ON-OFF pattern. Zeitler and Tass (2018) delivered PPMS at the frequency of the dominant synchronous rhythm using model stimuli that represented excitatory postsynaptic potentials, i.e., similar to Popovych and Tass (2012), as described in Supplementary Discussion 3.1. Synaptic weakening during PPMS was significantly weaker than during CR stimulation. They also reported entrainment between stimuli and spiking rhythms. In our network model, vPPMS neither caused acute nor longlasting desynchronization.

The poor performance of vPPMS as compared to vCR stimulation indicates that separate stimulation of different neuronal subpopulations is critical for desynchronization. The latter corresponds to the originally proposed desynchronization mechanism of CR stimulation (Tass, 2003). vPPMS, however, did not destabilize the pathological synchronous rhythm but caused an entrainment with the stimulus pattern (Figure 9).

We found that the distribution of spike times during collective spiking events broadened during stimulation $\mathrm{ON}$ periods of vPPMS, whereas it sharpened again during stimulation OFF periods due to synaptic interactions (Figure 9). We speculate that uninterrupted vPPMS, i.e., delivered without OFF periods, may cause desynchronization by further broadening collective spiking events until these events disappear and the system desynchronizes. 
vPPMS did not reduce synaptic weights. Instead, we found a slight increase of the mean synaptic weight in most parts of the parameter space (Figure 11C). This resulted from broad distributions of spike times during collective spiking events; see above. For strong vPPMS, synaptic weights increased substantially (Figure 11C). This increase was not observed by Zeitler and Tass (2018) probably because they considered short phase resetting stimuli; see previous paragraph.

The comparison of our computational results to those of previous studies (Popovych and Tass, 2012; Zeitler and Tass, 2018; Kromer et al., 2020; Kromer and Tass, 2020) indicates that the statistics of spiking responses to individual stimuli may have a strong impact on the outcome of stimulation. While model stimuli of previous studies led to a phase reset (Popovych and Tass, 2012; Zeitler and Tass, 2018; Kromer et al., 2020; Kromer and Tass, 2020), our vibrotactile model stimuli caused multiple collective spiking events and did not necessarily result in a phase reset of the collective rhythm. Nevertheless, vCR stimulation yielded pronounced acute and long-lasting desynchronization effects. This provides evidence that desynchronization effects of CR stimulation may not rely on phase-resetting stimuli and be more robust as originally assumed. In future studies, we intend to study the impact of the stimulus type on long-lasting desynchronization effects in more detail.

\section{Comparison Between Clinical and Computational Results}

In both our clinical and our computational studies, vCR stimulation entailed pronounced long-lasting desynchronization of neuronal activity.

\section{Measures of Synchrony}

In our clinical studies, neuronal synchrony was quantified using EEG data of the sensorimotor cortex. High EEG power is typically associated with synchronous activity of a large population of neurons in the corresponding frequency band (Musall et al., 2014). However, it is generally assumed that the EEG is generated by postsynaptic potentials in neurons near the cortical surface rather than action potentials (Ebersole and Milton, 2003).

In our computational study, we measured neuronal synchrony using the Kuramoto order parameter. The latter measures the degree of in-phase synchronized spiking activity. Low values of the Kuramoto order parameter can correspond either to desynchronized neuronal activity or to partially synchronized states, such as cluster states (Tass, 1999). Our computational model possessed a rather limited set of stable dynamical states, i.e., desynchronized and synchronized states, that could be adequately distinguished using the Kuramoto order parameter. However, the latter may not be sufficient to distinguish between more complex dynamical states, such as multiple interacting rhythms (Hyafil et al., 2015) or chimera states comprising portions of synchronized and desynchronized neurons (Majhi et al., 2019).

In PD patients, synchronized activity in different frequency bands is related to different symptoms. Synchronized activity in the theta band $(3-10 \mathrm{~Hz})$ has been associated with symptoms such as dyskinesia and tremor (Brown, 2003; Steigerwald et al., 2008; Tass et al., 2010; Contarino et al., 2012) and synchronized activity in the beta band (13-30 Hz) with rigidity and bradykinesia (Kühn et al., 2006; Weinberger et al., 2006). Therefore, it is likely that different rhythms interact in PDrelated brain networks. However, it is unclear whether chimera states occur in PD-related brain networks. In future studies, we anticipate considering more complex network models that allow us to study the influence of vibrotactile stimulation on networks with pathological synchrony in different frequency bands.

\section{Effects of Vibrotactile Coordinated Reset Stimulation Build Up Slowly}

In both our clinical and computational studies, effects of vCR stimulation built up slowly. In clinical study 1, we observed a significant improvement of the MDS-UPDRS III after $2 \mathrm{~h}$ of stimulation and a drop in high beta power over the sensorimotor cortex after 3 months of daily vCR stimulation, whereas no significant decrease of beta power was observed during and shortly after a 10-min vCR stimulation session during the first visit. This slow onset of stimulation effects was in marked contrast to classical DBS, where symptoms are suppressed within seconds to minutes after stimulation onset and resembled the slow buildup of effects observed in CR-DBS delivered to the subthalamic nucleus in PD patients (Adamchic et al., 2014). In our computational model, we observed an immediate reduction of the Kuramoto order parameter, corresponding to a reduction of neuronal synchrony (Figure 10). On the other hand, stimulation only slowly reduced synaptic weights. However, a sufficient reduction of the latter is required to drive the network into the attractor of a stable desynchronized and cause long-lasting desynchronization effects that outlast stimulation. Thus, while our model predicted a reduction of neuronal synchrony shortly after stimulation onset, which we did not observe in our clinical study, these effects only persisted after cessation of stimulation if a sufficient reduction of synaptic weights was achieved.

\section{Regular Coordinated Reset and Noisy Coordinated Reset Lead to Similar Results}

Neither our clinical nor our computational studies revealed qualitative differences between effects of regular and noisy vCR stimulation. While the number of patients considered in our clinical feasibility studies was too small to draw strong conclusions, our computational results created promising evidence that the effects of vCR stimulation are robust with respect to the considered stochastic variation of stimulusonset times. In our neuronal network model, both regular and noisy vCR stimulation had pronounced acute and long-lasting desynchronization effects. These effects were most pronounced if more than one stimulus per cycle of the synchronous rhythm was delivered and stimulation was strong enough to drive neurons over the spiking threshold, such that it provides control over the neuronal spiking activity. Regular and noisy CR may have similar effects due to variable neuronal responses to vibrotactile stimuli. Unlike a sharp phase reset, vibrotactile stimuli caused several variable collective spiking events of the stimulated neuronal subpopulations. This might have masked the 
additional stimulus delivery time jitter, which was constrained to avoid vibrotactile masking of subsequent stimuli (Hollins et al., 1990; Figure 9). Note that vPPMS caused neither acute nor long-lasting desynchronization (Figure 11), which indicates that separate stimulation of neuronal subpopulations is essential for desynchronization effects.

\section{Limitations and Future Directions}

An interesting prediction of our computational model is that vCR predominantly weakens synapses that interconnect neuronal subpopulation that respond to stimuli delivered to different fingertips. It is however unclear how synapses between stimulated and not stimulated subpopulations are affected. In our clinical study, we observed a reduction of beta power over a wide region of the sensorimotor cortex (Figure 7) and a reduction of the MDS-UPDRS III, indicating that therapeutic effects of vCR stimulation were not limited to brain regions responding to vibrotactile stimulation of the fingertips. In order to understand the widespread therapeutic effects of vCR fingertip stimulation, we intend to address the propagation of desynchronization effects between different neuronal subnetworks in future studies.

\section{Proprioceptive Path to Motor Circuits and Beyond}

During stimulation, vibrotactile bursts were delivered to the fingertips. Following the somatosensory pathway, highfrequency sinusoidal vibratory stimuli evoked strong spiking responses of thalamic neurons in the somatosensory thalamic nucleus [Vc (ventral caudal)] that are related to fast-adapting Pacinian corpuscle (PC) mechanoreceptors (Weiss et al., 2009). Furthermore, a substantial increase in the firing rates and pronounced phase locking of neuronal activity to vibratory stimuli was observed. Tactile Vc neurons relay sensory input to the somatosensory cortex SI. Experiments in macaque monkeys showed that sinusoidal high-frequency vibrotactile stimulation causes phase-locked spiking responses of SI neurons (Harvey et al., 2013). The authors also observed a logarithmic increase of the neurons' mean firing rate with the stimulation amplitude.

The complex interconnectivity of cortical areas is to date not completely understood. It is therefore not known how cortical activity patterns in SI propagate to motor areas exhibiting pathological oscillations in PD (Lindenbach and Bishop, 2013). It is possible that propagation occurs via projections from somatosensory cortical areas to the motor cortex. Such connections have been observed in mammals across species (Jones and Powell, 1968; Jones and Powell, 1969; Mao et al., 2011). Furthermore, experimental studies reported that some thalamic neurons in the cat sensory nucleus ventralis posterolateralis and corresponding nuclei in monkeys project directly to the motor cortex [see Asanuma and Mackel (1989) for a review].

We found long-lasting changes of cortical activity in PD patients after vCR stimulation (Figure 7). Previous studies reported a long-lasting reorganization of motor areas in response to tetanic stimulation of the somatosensory cortex (Iriki et al., 1989; Keller et al., 1990). Another study reported that sensory stimulation of the pharynx also evokes long-lasting changes of excitability and the organization of the swallowing motor cortex (Hamdy et al., 1998). These changes were shown to outlast stimulation for at least $30 \mathrm{~min}$, indicating that sensory stimulation can cause long-lasting changes in the motor cortex.

vCR stimulation may also activate proprioceptive pathways. Proprioceptive feedback is, for instance, provided by both muscle spindle afferents (Goodwin et al., 1972) and cutaneous receptors in the skin (Edin and Abbs, 1991; Collins et al., 2005). The frequency response of muscle-sensitive proprioceptive receptors is typically limited to approximately $120 \mathrm{~Hz}$ (Burke et al., 1976; Roll and Vedel, 1982) but may rise to $220 \mathrm{~Hz}$ for the most sensitive receptors (Burke et al., 1976; Roll et al., 1989). However, the frequency response of cutaneous receptors can be as high as $280 \mathrm{~Hz}$ (Ribot-Ciscar et al., 1989). In our clinical studies presented here, we used vibratory frequencies of $250 \mathrm{~Hz}$, which may have led to a predominant activation of cutaneous receptors. Note, Lee et al. (2012) show results at $250 \mathrm{~Hz}$ with C-2 tactors (i.e., very similar mechanical stimulators used in this study) that indicate proprioceptive receptor response (postural).

Proprioceptive feedback during voluntary movements has been found to diminish parkinsonian tremor (Naros et al., 2018). Furthermore, thalamic neurons in the ventral intermediate nucleus (Vim), a target area for chronic deep brain stimulation as a treatment of tremor-dominant PD (Benabid et al., 1996), respond to proprioceptive stimulation (El-Tahawy et al., 2004). Evaluating tremor suppression for different electrode locations within the Vim, Milosevic et al. (2018) found that stimulation sites near the border between the Vim and the Vc lead to most efficient tremor reduction. Neurons in that border region respond to somatic inputs arising in muscle, joints, and deep tissue (Vitek et al., 1994; El-Tahawy et al., 2004). Milosevic et al. (2018) suggested that the stimulation-induced interruption of the pacing of proprioceptive input may contribute to tremor suppression.

Diminished amounts of brain-derived neurotrophic factor (BDNF) have been found in cerebrospinal fluid (CSF) within the ventricular or lumbar regions, autopsy reports of $\mathrm{PD}$ patient brains, and in Parkinson disease-induced [1-methyl-4-phenyl1,2,3,6-tetrahydropyridine (MPTP) and 6-hydroxydopamine (6OHDA)] animal models (Nagatsu and Sawada, 2005). This decrease in BDNF has been associated with dopamine depletion in the substantia nigra pars compacta and destruction of presynaptic terminals in addition to dopamine shortages that account for the majority of movement abnormalities in PD individuals (Nagatsu et al., 2000). BDNF is an abundant "neuroprotectant" protein found in the hippocampus and cerebral cortices (Deogracias et al., 2012). BDNF is delivered to the striatal area from the hippocampus and cerebral cortex (i.e., afferent pathway; Baquet et al., 2004). This nerve growth factor enhances growth of all affected neurons in PD (Zuccato and Cattaneo, 2007). Vibrational stimulation likely utilizes a similar pathway and may provide therapeutic PD benefits via upregulating BDNF resulting in increases in dopaminergic neurons. This hypothesis has been tested in a previous study of MPTP-treated mice showing that 4 weeks of low-amplitude $(5 \mathrm{~mm}$ ) vibrational therapy (where mice were freely roaming on a vibrational platform) significantly increased the amount of nigrostriatal dopaminergic neurons and quantity of BDNF 
(Zhao et al., 2014). This may suggest that vibrational therapy could have a beneficial dopaminergic effect on the nigrostriatal pathway within the PD brain. One might hypothesize that roaming-related sequences of vibrational bursts could exert desynchronizing effects on motor circuits. In that case, diseasemodifying effects of vibrational therapy might possibly resemble those of STN-DBS observed in several animal studies (Chen et al., 2000; Maesawa et al., 2004; Wallace et al., 2007; Khaindrava et al., 2011; Spieles-Engemann et al., 2011; Musacchio et al., 2017), e.g., attributed to a reduction of glutamatergic excitotoxicity of the substantia nigra pars compacta (Chen et al., 2000; Maesawa et al., 2004; Nakao et al., 1999; Piallat et al., 1996; Wallace et al., 2007).

\section{CONCLUSION}

Our clinical and computational results illustrate promising therapeutic effects of vCR therapy. Clinically, PD patients exhibited sustained cumulative benefits from continued used of $\mathrm{vCR}$ treatment. In conclusion, the results presented here demonstrate the feasibility and preliminary efficacy of $\mathrm{vCR}$, which will properly enable us to plan a proof-of-concept study.

\section{DATA AVAILABILITY STATEMENT}

The original contributions presented in the study are included in the article/Supplementary Material, further inquiries can be directed to the corresponding author.

\section{ETHICS STATEMENT}

The studies involving human participants were reviewed and approved by Stanford Institutional Review Board. The patients/participants provided their written informed consent to participate in this study. Written informed consent was obtained from the individuals for the publication of any potentially identifiable images or data included in this article.

\section{REFERENCES}

Abbott, L. F., and Nelson, S. B. (2000). Synaptic plasticity: taming the beast. Nat. Neurosci. 3, 1178-1183. doi: 10.1038/81453

Adamchic, I., Hauptmann, C., Barnikol, U. B., Pawelczyk, N., Popovych, O., Barnikol, T. T., et al. (2014). Coordinated reset neuromodulation for Parkinson's disease: proof-of-concept study. Mov. Disord. 29, 1679-1684. doi: $10.1002 / \mathrm{mds} .25923$

Asanuma, H., and Mackel, R. (1989). Direct and indirect sensory input pathways to the motor cortex; its structure and function in relation to learning of motor skills. Japn. J. Physiol. 39, 1-19. doi: 10.2170/jjphysiol.39.1

Baquet, Z. C., Gorski, J. A., and Jones, K. R. (2004). Early striatal dendrite deficits followed by neuron loss with advanced age in the absence of anterograde cortical brain-derived neurotrophic factor. J. Neurosci. 24, 4250-4258. doi: 10.1523/jneurosci.3920-03.2004

\section{AUTHOR CONTRIBUTIONS}

JK and PT designed the computational study and analyzed computational data. JK performed the simulations. $\mathrm{KP}, \mathrm{RD}, \mathrm{CH}$, and PT designed the clinical study protocol. KP, AC, TH, EL, $\mathrm{AF}, \mathrm{CH}$, and $\mathrm{PT}$ performed the clinical exams and/or took care of patients. $\mathrm{KP}, \mathrm{SH}$, and $\mathrm{PT}$ contributed to clinical data analysis. KP and AC performed EEG recordings. KP, AC, and $\mathrm{PT}$ analyzed the EEG data. BM and PT designed the vibrotactile glove system. KP, JK, and PT wrote the manuscript. All authors revised/approved the manuscript.

\section{FUNDING}

We gratefully acknowledge funding support by The Anonymous Life Sciences Fund, McGrath Family Foundation, John A. Blume Foundation, and Parkinson Alliance vCR Fund. We are also grateful to the Binns Family Foundation for support of our EEG Neuromodulation lab.

\section{ACKNOWLEDGMENTS}

Some of the computing for this project was performed on the Sherlock cluster. We would like to thank Stanford University and the Stanford Research Computing Center for providing computational resources and support that contributed to these research results. Additionally, we gratefully acknowledge discussions with Dr. Roger Cholewiak and François Tadel. We thank Dr. David Riley for administering the MDS-UPDRS III in one of our patients due to COVID-19-related restrictions. We thank Dr. Tina Munjal for helpful comments on our manuscript. We thank Synergic Medical Technologies, Inc. for supplying us with glove systems.

\section{SUPPLEMENTARY MATERIAL}

The Supplementary Material for this article can be found online at: https://www.frontiersin.org/articles/10.3389/fphys. 2021.624317/full\#supplementary-material

Benabid, A. L., Pollak, P., Gao, D., Hoffmann, D., Limousin, P., Gay, E., et al. (1996). Chronic electrical stimulation of the ventralis intermedius nucleus of the thalamus as a treatment of movement disorders. J. Neurosurg. 84, 203-214. doi: 10.3171/jns.1996.84.2.0203

Bi, G. Q., and Poo, M. M. (1998). Synaptic modifications in cultured hippocampal neurons: dependence on spike timing, synaptic strength, and postsynaptic cell type. J. Neurosci. 18, 10464-10472. doi: 10.1523/jneurosci.18-24-10464. 1998

Bronte-Stewart, H., Barberini, C., Koop, M. M., Hill, B. C., Henderson, J. M., and Wingeier, B. (2009). The STN beta-band profile in Parkinson's disease is stationary and shows prolonged attenuation after deep brain stimulation. Exp. Neurol. 215, 20-28. doi: 10.1016/j.expneurol.2008.09.008

Brown, P. (2003). Oscillatory nature of human basal ganglia activity: relationship to the pathophysiology of Parkinson's disease. Mov. Disord. 18, 357-363. doi: $10.1002 / \mathrm{mds} .10358$ 
Brown, P., Oliviero, A., Mazzone, P., Insola, A., Tonali, P., and Di Lazzaro, V. (2001). Dopamine dependency of oscillations between subthalamic nucleus and pallidum in Parkinson's disease. J. Neurosci. 21, 1033-1038. doi: 10.1523/ jneurosci.21-03-01033.2001

Burke, D., Hagbarth, K. E., Löfstedt, L., and Wallin, B. G. (1976). The responses of human muscle spindle endings to vibration of non-contracting muscles. J. Physiol. 261, 673-693. doi: 10.1113/jphysiol.1976.sp011580

Caporale, N., and Dan, Y. (2008). Spike timing-dependent plasticity: a Hebbian learning rule. Ann. Rev. Neurosci. 31, 25-46. doi: 10.1146/annurev.neuro.31. 060407.125639

Chen, L., Liu, Z., Tian, Z., Wang, Y., and Li, S. (2000). Prevention of neurotoxin damage of 6-OHDA to dopaminergic nigral neuron by subthalamic nucleus lesions. Stereotact. Funct. Neurosurg. 75, 66-75. doi: 10.1159/0000 48385

Collins, D. F., Refshauge, K. M., Todd, G., and Gandevia, S. C. (2005). Cutaneous receptors contribute to kinesthesia at the index finger, elbow, and knee. J. Neurophysiol. 94, 1699-1706. doi: 10.1152/jn.00191.2005

Contarino, M. F., Bour, L. J., Bot, M., van den Munckhof, P., Speelman, J. D., Schuurman, P. R., et al. (2012). Tremor-specific neuronal oscillation pattern in dorsal subthalamic nucleus of parkinsonian patients. Brain Stimul. 5, 305-314. doi: 10.1016/j.brs.2011.03.011

Crabtree, J. W. (2018). Functional diversity of thalamic reticular subnetworks. Front. Syst. Neurosci. 12:41. doi: 10.3389/fnsys.2018.00041

Crabtree, J. W., and Isaac, J. T. R. (2002). New intrathalamic pathways allowing modality-related and cross-modality switching in the dorsal thalamus. J. Neurosci. 22, 8754-8761. doi: 10.1523/jneurosci.22-19-08754.2002

Craig, J. C. (1985). Attending to two fingers: two hands are better than one. Percept. Psychophys. 38, 496-511. doi: 10.3758/bf03207059

Craig, J. C., and Qian, X. (1997). Tactile pattern perception by two fingers: temporal interference and response competition. Percept. Psychophys. 59, 252-265. doi: 10.3758/bf03211893

Delorme, A., and Makeig, S. (2004). EEGLAB: an open source toolbox for analysis of single-trial EEG dynamics including independent component analysis. J. Neurosci. Methods 134, 9-21. doi: 10.1016/j.jneumeth.2003.10.009

Deogracias, R., Yazdani, M., Dekkers, M. P. J., Guy, J., Ionescu, M. C. S., Vogt, K. E., et al. (2012). Fingolimod, a sphingosine-1 phosphate receptor modulator, increases BDNF levels and improves symptoms of a mouse model of Rett syndrome. Proc. Natl. Acad. Sci. U.S.A. 109, 14230-14235. doi: 10.1073/pnas. 1206093109

Dincher, A., Schwarz, M., and Wydra, G. (2019). Analysis of the effects of whole-body vibration in parkinson disease-systematic review and meta-analysis. PM R 11, 640-653. doi: 10.1002/pmrj.12094

Ebersbach, G., Edler, D., Kaufhold, O., and Wissel, J. (2008). Whole body vibration versus conventional physiotherapy to improve balance and gait in Parkinson's disease. Arch. Phys. Med. Rehabil. 89, 399-403. doi: 10.1016/j.apmr.2007.09.031

Ebersole, J. S., and Milton, J. (2003). "The electroencephalogram (EEG): a measure of neural synchrony," in Epilepsy as a Dynamic Disease, eds J. Milton and P. Jung (Berlin: Springer), 51-68. doi: 10.1007/978-3-662-05048-4_5

Edin, B. B., and Abbs, J. H. (1991). Finger movement responses of cutaneous mechanoreceptors in the dorsal skin of the human hand. J. Neurophysiol. 65 , 657-670. doi: 10.1152/jn.1991.65.3.657

El-Tahawy, H., Lozano, A. M., and Dostrovsky, J. O. (2004). "Electrophysiological findings in Vim and Vc," in Microelectrode Recording in Movement Disorder Surgery, ed. S. Liu (New York, NY: Thieme Medical Publishers, Inc.), 63-71.

Espay, A. J., Fasano, A., Van Nuenen, B. F. L., Payne, M. M., Snijders, A. H., and Bloem, B. R. (2012). "On" state freezing of gait in Parkinson disease: a paradoxical levodopa-induced complication. Neurology 78, 454-457. doi: 10. 1212/wnl.0b013e3182477ec0

Flaherty, A. W., and Graybiel, A. M. (1991). Corticostriatal transformations in the primate somatosensory system. Projections from physiologically mapped bodypart representations. J. Neurophysiol. 66, 1249-1263. doi: 10.1152/jn.1991.66.4. 1249

Gescheider, G. A., Berryhill, M. E., Verrillo, R. T., and Bolanowski, S. J. (1999). Vibrotactile temporal summation: probability summation or neural integration? Somatosens. Motor Res. 16, 229-242. doi: 10.1080/08990229970483

Giannicola, G., Marceglia, S., Rossi, L., Mrakic-Sposta, S., Rampini, P., Tamma, F., et al. (2010). The effects of levodopa and ongoing deep brain stimulation on subthalamic beta oscillations in Parkinson's disease. Exp. Neurol. 226, 120-127. doi: 10.1016/j.expneurol.2010.08.011

Goetz, C. G., Leurgans, S., Raman, R., and Stebbins, G. T. (2000). Objective changes in motor function during placebo treatment in PD. Neurology 54, 710-714. doi: 10.1212/wnl.54.3.710

Goetz, C. G., Tilley, B. C., Shaftman, S. R., Stebbins, G. T., Fahn, S., Martinez-Martin, P., et al. (2008a). Movement disorder society-sponsored revision of the unified Parkinson's disease rating scale (MDS-UPDRS): scale presentation and clinimetric testing results. Mov. Dis. 23, 2129-2170. doi: $10.1002 / \mathrm{mds} .22340$

Goetz, C. G., Wuu, J., McDermott, M. P., Adler, C. H., Fahn, S., Freed, C. R., et al. (2008b). Placebo response in Parkinson's disease: comparisons among 11 trials covering medical and surgical interventions. Mov. Disord. 23, 690-699. doi: $10.1002 / \mathrm{mds} .21894$

Goodwin, A. W. (2005). Paradoxes in tactile adaptation. focus on "Vibratory adaptation in cutaneous mechanoreceptive afferents" and "Time-course of vibratory adaptation and recovery in cutaneous mechanoreceptive afferents". J. Neurophysiol. 94, 2995-2996. doi: 10.1152/jn.00766.2005

Goodwin, G. M., McCloskey, D. I., and Matthews, P. B. C. (1972). The contribution of muscle afferents to kinaesthesia shown by vibration induced illusions of movement and by the effects of paralysing joint afferents. Brain 95, 705-748. doi: 10.1093/brain/95.4.705

Green, B. G. (1976). Vibrotactile temporal summation: effect of frequency. Sens. Process. 1, 138-149.

Haas, C. T., Turbanski, S., Kessler, K., and Schmidtbleicher, D. (2006). The effects of random whole-body-vibration on motor symptoms in Parkinson's disease. NeuroRehabilitation 21, 29-36. doi: 10.3233/nre-2006-21105

Hahn, J. F. (1968). Low-frequency vibrotactile adaptation. J. Exp. Psychol. 78(4 Pt 1), 655-659. doi: 10.1037/h0026621

Hamdy, S., Rothwell, J. C., Aziz, Q., Singh, K. D., and Thompson, D. G. (1998). Long-term reorganization of human motor cortex driven by short-term sensory stimulation. Nat. Neurosci. 1, 64-68. doi: 10.1038/264

Hammond, C., Bergman, H., and Brown, P. (2007). Pathological synchronization in Parkinson's disease: networks, models and treatments. Trends Neurosci. 30, 357-364. doi: 10.1016/j.tins.2007.05.004

Hartmann-von Monakow, K., Akert, K., and Künzle, H. (1978). Projections of the precentral motor cortex and other cortical areas of the frontal lobe to the subthalamic nucleus in the monkey. Exp. Brain Res. 33, 395-403.

Harvey, M. A., Saal, H. P., Dammann, J. F. III, and Bensmaia, S. J. (2013). Multiplexing stimulus information through rate and temporal codes in primate somatosensory cortex. PLoS Biol. 11:e1001558. doi: 10.1371/journal.pbio. 1001558

Hauptmann, C., and Tass, P. A. (2009). Cumulative and after-effects of short and weak coordinated reset stimulation: a modeling study. J. Neural Eng. 6:016004. doi: 10.1088/1741-2560/6/1/016004

Holden, S. K., Finseth, T., Sillau, S. H., and Berman, B. D. (2018). Progression of MDS-UPDRS scores over five years in de novo Parkinson disease from the Parkinson's progression markers initiative cohort. Mov. Dis. Clin. Pract. 5, 47-53. doi: $10.1002 / \mathrm{mdc} 3.12553$

Hollins, M., Goble, A. K., Whitsel, B. L., and Tommerdahl, M. (1990). Time course and action spectrum of vibrotactile adaptation. Somatosens. Motor Res. 7, 205-221. doi: 10.3109/08990229009 144707

Horváth, K., Aschermann, Z., Ács, P., Deli, G., Janszky, J., Komoly, S., et al. (2015). Minimal clinically important difference on the Motor examination part of MDS-UPDRS. Parkinsonism Relat. Disord. 21, 1421-1426. doi: 10.1016/j. parkreldis.2015.10.006

Hyafil, A., Giraud, A. L., Fontolan, L., and Gutkin, B. (2015). Neural crossfrequency coupling: connecting architectures, mechanisms, and functions. Trends Neurosci. 38, 725-740. doi: 10.1016/j.tins.2015.09.001

Hyvarinen, A. (1999). Fast and robust fixed-point algorithms for independent component analysis. IEEE Trans. Neural Netw. 10, 626-634. doi: 10.1109/72. 761722

Iriki, A., Pavlides, C., Keller, A., and Asanuma, H. (1989). Long-term potentiation in the motor cortex. Science 245, 1385-1387. doi: 10.1126/science.2551038

Jenkinson, C., Fitzpatrick, R., Peto, V., Greenhall, R., and Hyman, N. (1997). The Parkinson's disease questionnaire (PDQ-39): development and validation 
of a Parkinson's disease summary index score. Age Ageing 26, 353-357. doi: 10.1093/ageing/26.5.353

Johansson, R. S., and Vallbo, ÅB. (1983). Tactile sensory coding in the glabrous skin of the human hand. Trends Neurosci. 6, 27-32. doi: 10.1016/0166-2236(83) 90011-5

Jones, E. G., and Powell, T. P. S. (1968). The commissural connexions of the somatic sensory cortex in the cat. J. Anat. 103(Pt 3), 433-455.

Jones, E. G., and Powell, T. P. S. (1969). Connexions of the somatic sensory cortex of the rhesus monkey: I.-Ipsilateral cortical connexions. Brain 92, 477-502. doi: 10.1093/brain/92.3.477

Kaneko, T., Caria, M. A., and Asanuma, H. (1994). Information processing within the motor cortex. I. Responses of morphologically identified motor cortical cells to stimulation of the somatosensor cortex. J. Comp. Neurol. 345, 161-171. doi: 10.1002/cne.903450202

Keller, A., Iriki, A., and Asanuma, H. (1990). Identification of neurons producing long-term potentiation in the cat motor cortex: intracellular recordings and labeling. J. Comp. Neurol. 300, 47-60. doi: 10.1002/cne.903000105

Khaindrava, V., Salin, P., Melon, C., Ugrumov, M., Kerkerian-Le-Goff, L., and Daszuta, A. (2011). High frequency stimulation of the subthalamic nucleus impacts adult neurogenesis in a rat model of Parkinson's disease. Neurobiol. Dis. 42, 284-291. doi: 10.1016/j.nbd.2011.01.018

King, L. K., Almeida, Q. J., and Ahonen, H. (2009). Short-term effects of vibration therapy on motor impairments in Parkinson's disease. NeuroRehabilitation 25, 297-306. doi: 10.3233/nre-2009-0528

Kita, T., Shigematsu, N., and Kita, H. (2016). Intralaminar and tectal projections to the subthalamus in the rat. Eur. J. Neurosci. 44, 2899-2908. doi: 10.1111/ejn. 13413

Knibestöl, M. (1973). Stimulus-response functions of rapidly adapting mechanoreceptors in the human glabrous skin area. J. Physiol. 232, 427-452. doi: 10.1113/jphysiol.1973.sp010279

Knoblauch, A., Hauser, F., Gewaltig, M.-O., Körner, E., and Palm, G. (2012). Does spike-timing-dependent synaptic plasticity couple or decouple neurons firing in synchrony? Front. Comput. Neurosci. 6:55. doi: 10.3389/fncom.2012. 00055

Kromer, J. A., Khaledi-Nasab, A., and Tass, P. A. (2020). Impact of number of stimulation sites on long-lasting desynchronization effects of coordinated reset stimulation. Chaos 30:083134. doi: 10.1063/5.0015196

Kromer, J. A., and Tass, P. A. (2020). Long-lasting desynchronization by decoupling stimulation. Phys. Rev. Res. 2:033101

Kuramoto, Y. (1984). Chemical Oscillations, Waves, and Turbulence. Berlin: Springer.

Kühn, A. A., Kupsch, A., Schneider, G.-H., and Brown, P. (2006). Reduction in subthalamic 8-35 hz oscillatory activity correlates with clinical improvement in parkinson's disease. Eur. J. Neurosci. 23, 1956-1960. doi: 10.1111/j.1460-9568. 2006.04717.x

Lau, R. W. K., Teo, T., Yu, F., Chung, R. C. K., and Pang, M. Y. C. (2011). Effects of whole-body vibration on sensorimotor performance in people with Parkinson disease: a systematic review. Phys. Ther. 91, 198-209. doi: 10.2522/ptj.201 00071

Lee, B.-C., Martin, B. J., and Sienko, K. H. (2012). Directional postural responses induced by vibrotactile stimulations applied to the torso. Exp. Brain Rres. 222, 471-482. doi: 10.1007/s00221-012-3233-2

Leung, Y. Y., Bensmaïa, S. J., Hsiao, S. S., and Johnson, K. O. (2005). Timecourse of vibratory adaptation and recovery in cutaneous mechanoreceptive afferents. J. Neurophysiol. 94, 3037-3045. doi: 10.1152/jn.00001. 2005

Li, X., Xing, Y., Martin-Bastida, A., Piccini, P., and Auer, D. P. (2018). Patterns of grey matter loss associated with motor subscores in early Parkinson's disease. Neuroimage Clin. 17, 498-504. doi: 10.1016/j.nicl.2017.11.009

Lindenbach, D., and Bishop, C. (2013). Critical involvement of the motor cortex in the pathophysiology and treatment of Parkinson's disease. Neurosci. Biobehav. Rev. 37, 2737-2750. doi: 10.1016/j.neubiorev.2013.09.008

López-Azcárate, J., Tainta, M., Rodríguez-Oroz, M. C., Valencia, M., González, R., Guridi, J., et al. (2010). Coupling between beta and high-frequency activity in the human subthalamic nucleus may be a pathophysiological mechanism in Parkinson's disease. J. Neurosci. 30, 6667-6677. doi: 10.1523/jneurosci.545909.2010
Lubenov, E. V., and Siapas, A. G. (2008). Decoupling through synchrony in neuronal circuits with propagation delays. Neuron 58, 118-131. doi: 10.1016/j. neuron.2008.01.036

Ly, Q. T., Handojoseno, A. M. A., Gilat, M., Nguyen, N., Chai, R., Tran, Y., et al. (2016). "Detection of gait initiation failure in Parkinson's disease patients using EEG signals," in Proceedings of the $201638^{\text {th }}$ Annual International Conference of the IEEE Engineering in Medicine and Biology Society (EMBC), (Orlando, FL: IEEE), 1599-1602.

Lysyansky, B., Popovych, O. V., and Tass, P. A. (2011). Desynchronizing antiresonance effect of m: n ON-OFF coordinated reset stimulation. J. Neural Eng. 8:036019. doi: 10.1088/1741-2560/8/3/036019

Maesawa, S., Kaneoke, Y., Kajita, Y., Usui, N., Misawa, N., Nakayama, A., et al. (2004). Long-term stimulation of the subthalamic nucleus in hemiparkinsonian rats: neuroprotection of dopaminergic neurons. J. Neurosurg. 100, 679-687. doi: 10.3171/jns.2004.100.4.0679

Majhi, S., Bera, B. K., Ghosh, D., and Perk, M. (2019). Chimera states in neuronal networks: a review. Phys. Life Rev. 28, 100-121. doi: 10.1016/j.plrev.2018. 09.003

Mao, T., Kusefoglu, D., Hooks, B. M., Huber, D., Petreanu, L., and Svoboda, K. (2011). Long-range neuronal ciruits underlying the interaction between sensory and motor cortex. Neuron 71, 111-123. doi: 10.1016/j.neuron.2011.07.029

Marín, P. J., Herrero, A. J., Milton, J. G., Hazell, T. J., and García-López, D. (2013). Whole-body vibration applied during upper body exercise improves performance. J. Strength Cond. Res. 27, 1807-1812. doi: 10.1519/jsc. 0b013e3182772f00

Markram, H., Lübke, J., Frotscher, M., and Sakmann, B. (1997). Regulation of synaptic efficacy by coincidence of postsynaptic APs and EPSPs. Science 275, 213-215. doi: 10.1126/science.275.5297.213

Marsden, J. F., Limousin-Dowsey, P., Ashby, P., Pollak, P., and Brown, P. (2001). Subthalamic nucleus, sensorimotor cortex and muscle interrelationships in Parkinson's disease. Brain 124, 378-388. doi: 10.1093/brain/124.2.378

Milosevic, L., Kalia, S. K., Hodaie, M., Lozano, A. M., Popovic, M. R., and Hutchison, W. D. (2018). Physiological mechanisms of thalamic ventral intermediate nucleus stimulation for tremor suppression. Brain 141, 21422155. doi: 10.1093/brain/awy139

Mullen, T. R., Kothe, C. A. E., Chi, Y. M., Ojeda, A., Kerth, T., Makeig, S., et al. (2015). Real-time neuroimaging and cognitive monitoring using wearable dry EEG. IEEE Trans. Biomed. Eng. 62, 2553-2567. doi: 10.1109/tbme.2015. 2481482

Musacchio, T., Rebenstorff, M., Fluri, F., Brotchie, J. M., Volkmann, J., Koprich, J. B., et al. (2017). Subthalamic nucleus deep brain stimulation is neuroprotective in the A53T $\alpha$-synuclein Parkinson's disease rat model. Ann. Neurol. 81, 825-836. doi: 10.1002/ana.24947

Musall, S., von Pföstl, V., Rauch, A., Logthetis, N. K., and Whittingstall, K. (2014). Effects of neural synchrony on surface EEG. Cereb. Cortex 24, 1045-1053. doi: 10.1093/cercor/bhs389

Nagatsu, T., Mogi, M., Ichinose, H., and Togari, A. (2000). "Changes in cytokines and neurotrophins in Parkinson's disease," in Advances in Research on Neurodegeneration, eds P. Riederer, D. B. Calne, R. Horowski, Y. Mizuno, C. W. Olanow, W. Poewe, et al. (New York, NY: Springer), 277-290. doi: 10.1007/978-3-7091-6301-6_19

Nagatsu, T., and Sawada, M. (2005). Inflammatory process in Parkinson's disease: role for cytokines. Curr. Pharm. Design 11, 999-1016. doi: 10.2174/ 1381612053381620

Nakao, N., Nakai, E., Nakai, K., and Itakura, T. (1999). Ablation of the subthalamic nucleus supports the survival of nigral dopaminergic neurons after nigrostriatal lesions induced by the mitochondrial toxin 3-nitropropionic acid. Ann. Neurol. 45, 640-651. doi: 10.1002/1531-8249(199905)45:5<640::aid-ana13>3. $0 . \mathrm{co} ; 2-\mathrm{u}$

Nambu, A., Takada, M., Inase, M., and Tokuno, H. (1996). Dual somatotopical representations in the primate subthalamic nucleus: evidence for ordered but reversed body-map transformations from the primary motor cortex and the supplementary motor area. J. Neurosci. 16, 2671-2683. doi: 10.1523/jneurosci. 16-08-02671.1996

Naros, G., Grimm, F., Weiss, D., and Gharabaghi, A. (2018). Directional communication during movement execution interferes with tremor in Parkinson's disease. Mov. Disord. 33, 251-261. doi: 10.1002/mds. 27221 
Neuper, C., and Pfurtscheller, G. (2001). Event-related dynamics of cortical rhythms: frequency-specific features and functional correlates. Int. J. Psychophysiol. 43, 41-58. doi: 10.1016/s0167-8760(01)00178-7

Neuper, C., Schlögl, A., and Pfurtscheller, G. (1999). Enhancement of leftright sensorimotor EEG differences during feedback-regulated motor imagery. J. Clin. Neurophysiol. 16, 373-382. doi: 10.1097/00004691-199907000-00010

Neuper, C., Wörtz, M., and Pfurtscheller, G. (2006). ERD/ERS patterns reflecting sensorimotor activation and deactivation. Prog. Brain Res. 159, 211-222. doi: 10.1016/s0079-6123(06)59014-4

Pascual-Marqui, R. D. (2002). Standardized low-resolution brain electromagnetic tomography (sLORETA): technical details. Methods Find. Exp. Clin. Pharmacol. 24(Suppl. D), 5-12.

Perrin, F., Pernier, J., Bertrand, O., and Echallier, J. F. (1989). Spherical splines for scalp potential and current density mapping. Electroencephalogr. Clin. Neurophysiol. 72, 184-187. doi: 10.1016/0013-4694(89)90180-6

Piallat, B., Benazzouz, A., and Benabid, A. L. (1996). Subthalamic nucleus lesion in rats prevents dopaminergic nigral neuron degeneration after striatal 6-OHDA injection: behavioural and immunohistochemical studies. Eur. J. Neurosci. 8, 1408-1414. doi: 10.1111/j.1460-9568.1996.tb01603.x

Popovych, O. V., and Tass, P. A. (2012). Desynchronizing electrical and sensory coordinated reset neuromodulation. Front. Hum. Neurosci. 6:58. doi: 10.3389/ fnhum.2012.00058

Powanwe, A. S., and Longtin, A. (2019). Determinants of brain rhythm burst statistics. Sci. Rep. 9:18335.

Priori, A., Foffani, G., Pesenti, A., Tamma, F., Bianchi, A. M., Pellegrini, M., et al. (2004). Rhythm-specific pharmacological modulation of subthalamic activity in Parkinson's disease. Exp. Neurol. 189, 369-379. doi: 10.1016/j.expneurol.2004. 06.001

Ribot-Ciscar, E., Vedel, J. P., and Roll, J. P. (1989). Vibration sensitivity of slowly and rapidly adapting cutaneous mechanoreceptors in the human foot and leg. Neurosci. Lett. 104, 130-135. doi: 10.1016/0304-3940(89)90342-x

Roll, J. P., and Vedel, J. P. (1982). Kinaesthetic role of muscle afferents in man, studied by tendon vibration and microneurography. Exp. Brain Res. 47, 177-190.

Roll, J. P., Vedel, J. P., and Ribot, E. (1989). Alteration of proprioceptive messages induced by tendon vibration in man: a microneurographic study. Exp. Brain Res. 76, 213-222.

Rosenblum, M., Pikovsky, A., Kurths, J., Schäfer, C., and Tass, P. A. (2001). "Phase synchronization: from theory to data analysis," in Handbook of Biological Physics, Vol. 4, eds F. Moss and S. Gielen (Amsterdam: Elsevier Science), 279-321. doi: 10.1016/s1383-8121(01)80012-9

Rosenkranz, K., and Rothwell, J. C. (2003). Differential effect of muscle vibration on intracortical inhibitory circuits in humans. J. Physiol. 551, 649-660. doi: 10.1113/jphysiol.2003.043752

Schaefer, A., Kong, R., Gordon, E. M., Laumann, T. O., Zuo, X.-N., Holmes, A. J., et al. (2018). Local-global parcellation of the human cerebral cortex from intrinsic functional connectivity MRI. Cereb. Cortex 28, 3095-3114. doi: 10. 1093/cercor/bhx179

Seo, N. J., Lakshminarayanan, K., Lauer, A. W., Ramakrishnan, V., Schmit, B. D., Hanlon, C. A., et al. (2019). Use of imperceptible wrist vibration to modulate sensorimotor cortical activity. Exp. Brain Res. 237, 805-816. doi: 10.1007/ s00221-018-05465-z

Spieles-Engemann, A. L., Steece-Collier, K., Behbehani, M. M., Collier, T. J., Wohlgenant, S. L., Kemp, C. J., et al. (2011). Subthalamic nucleus stimulation increases brain derived neurotrophic factor in the nigrostriatal system and primary motor cortex. J. Parkinsons Dis. 1, 123-136. doi: 10.3233/jpd-201111008

Stebbins, G. T., Goetz, C. G., Burn, D. J., Jankovic, J., Khoo, T. K., and Tilley, B. C. (2013). How to identify tremor dominant and postural instability/gait difficulty groups with the movement disorder society unified Parkinson's disease rating scale: comparison with the unified Parkinson's disease rating scale. Mov. Disord. 28, 668-670. doi: 10.1002/mds. 25383

Steigerwald, F., Pötter, M., Herzog, J., Pinsker, M., Kopper, F., Mehdorn, H., et al. (2008). Neuronal activity of the human subthalamic nucleus in the parkinsonian and nonparkinsonian state. J. Neurophysiol. 100, 2515-2524. doi: 10.1152/jn.90574.2008

Sugimoto, T., Hattori, T., Mizuno, N., Itoh, K., and Sato, M. (1983). Direct projections from the centre median-parafascicular complex to the subthalamic nucleus in the cat and rat. J. Comp. Neurol. 214, 209-216. doi: 10.1002/cne. 902140208

Syrkin-Nikolau, J., Neuville, R., O’Day, J., Anidi, C., Koop, M. M., Martin, T., et al. (2018). Coordinated reset vibrotactile stimulation shows prolonged improvement in Parkinson's disease. Mov. Disord. 33, 179-180. doi: 10.1002/ mds. 27223

Tadel, F., Baillet, S., Mosher, J. C., Pantazis, D., and Leahy, R. M. (2011). Brainstorm: a user-friendly application for MEG/EEG analysis. Comput. Intell. Neurosci. 2011:879716.

Tass, P., Smirnov, D., Karavaev, A., Barnikol, U., Barnikol, T., Adamchic, I., et al. (2010). The causal relationship between subcortical local field potential oscillations and Parkinsonian resting tremor. J. Neural Eng. 7:016009. doi: 10.1088/1741-2560/7/1/016009

Tass, P. A. (1999). Phase Resetting in Medicine and Biology: Stochastic Modelling and Data Analysis. Berlin: Springer.

Tass, P. A. (2003). A model of desynchronizing deep brain stimulation with a demand-controlled coordinated reset of neural subpopulations. Biol. Cybernet. 89, 81-88. doi: 10.1007/s00422-003-0425-7

Tass, P. A. (2017). Vibrotactile coordinated reset stimulation for the treatment of neurological diseases: concepts and device specifications. Cureus 9;e1535.

Tass, P. A., and Majtanik, M. (2006). Long-term anti-kindling effects of desynchronizing brain stimulation: a theoretical study. Biol. Cybernet. 94, 58-66. doi: 10.1007/s00422-005-0028-6

Tass, P. A., Qin, L., Hauptmann, C., Dovero, S., Bezard, E., Boraud, T., et al. (2012). Coordinated reset has sustained after effects in Parkinsonian monkeys. Ann. Neurol. 72, 816-820. doi: 10.1002/ana.23663

Toledo, J. B., López-Azcárate, J., Garcia-Garcia, D., Guridi, J., Valencia, M., Artieda, J., et al. (2014). High beta activity in the subthalamic nucleus and freezing of gait in Parkinson's disease. Neurobiol. Dis. 64, 60-65. doi: 10.1016/j.nbd.2013. 12.005

Tomlinson, C. L., Stowe, R., Patel, S., Rick, C., Gray, R., and Clarke, C. E. (2010). Systematic review of levodopa dose equivalency reporting in Parkinson's disease. Mov. Disord. 25, 2649-2653. doi: 10.1002/mds.23429

Verbaan, D., Jeukens-Visser, M., Van Laar, T., van Rooden, S. M., Van Zwet, E. W., Marinus, J., et al. (2011). SCOPA-cognition cutoff value for detection of Parkinson's disease dementia. Mov. Disord. 26, 1881-1886. doi: 10.1002/mds. 23750

Vitek, J. L., Ashe, J., DeLong, M. R., and Alexander, G. E. (1994). Physiologic properties and somatotopic organization of the primate motor thalamus. J. Neurophysiol. 71, 1498-1513. doi: 10.1152/jn.1994.71. 4.1498

Wallace, B. A., Ashkan, K., Heise, C. E., Foote, K. D., Torres, N., Mitrofanis, J., et al. (2007). Survival of midbrain dopaminergic cells after lesion or deep brain stimulation of the subthalamic nucleus in MPTP-treated monkeys. Brain 130, 2129-2145. doi: 10.1093/brain/awm137

Wang, J., Nebeck, S., Muralidharan, A., Johnson, M. D., Vitek, J. L., and Baker, K. B. (2016). Coordinated reset deep brain stimulation of subthalamic nucleus produces long-lasting, dose-dependent motor improvements in the 1-methyl-4-phenyl-1，2，3，6-tetrahydropyridine non-human primate model of parkinsonism. Brain Stimul. 9, 609-617. doi: 10.1016/j.brs.2016. 03.014

Weinberger, M., Mahant, N., Hutchison, W. D., Lozano, A. M., Moro, E., Hodaie, M., et al. (2006). Beta oscillatory activity in the subthalamic nucleus and its relation to dopaminergic response in Parkinson's disease. J. Neurophysiol. 96, 3248-3256. doi: 10.1152/jn.00697.2006

Weiss, N., Ohara, S., Johnson, K. O., and Lenz, F. A. (2009). The human thalamic somatic sensory nucleus [ventral caudal (Vc)] shows neuronal mechanoreceptor-like responses to optimal stimuli for peripheral mechanoreceptors. J. Neurophysiol. 101, 1033-1042. doi: $10.1152 /$ jn. 90990.2008

Whitmer, D., de Solages, C., Hill, B., Yu, H., Henderson, J. M., and Bronte-Stewart, H. (2012). High frequency deep brain stimulation attenuates subthalamic and cortical rhythms in Parkinson's disease. Front. Hum. Neurosci. 6:155. doi: 10. 3389/fnhum.2012.00155

Williams, D., Tijssen, M., Van Bruggen, G., Bosch, A., Insola, A., Di Lazzaro, V., et al. (2002). Dopamine-dependent changes in the functional connectivity between basal ganglia and cerebral cortex in humans. Brain 125, 1558-1569. doi: 10.1093/brain/awf156 
Zeitler, M., and Tass, P. A. (2015). Augmented brain function by coordinated reset stimulation with slowly varying sequences. Front. Syst. Neurosci. 9:49. doi: 10.3389/fnsys.2015.00049

Zeitler, M., and Tass, P. A. (2018). Computationally developed sham stimulation protocol for multichannel desynchronizing stimulation. Front. Physiol. 9:512. doi: 10.3389/fphys.2018.00512

Zhao, L., He, L. X., Huang, S. N., Gong, L. J., Li, L., Lv, Y. Y., et al. (2014). Protection of dopamine neurons by vibration training and up-regulation of brain-derived neurotrophic factor in a MPTP mouse model of Parkinson's disease. Physiol. Res. 63, 649-657. doi: 10.33549/physiolres.932743

Zuccato, C., and Cattaneo, E. (2007). Role of brain-derived neurotrophic factor in Huntington's disease. Prog. Neurobiol. 81, 294-330. doi: 10.1016/j.pneurobio. 2007.01.003

Conflict of Interest: TH works as consultant for Boston Scientific and received teaching honoraria for DBS courses. $\mathrm{RD}$ has served as a clinical trials investigator for Impax Pharmaceuticals, Pharma2B, CALA Health, Axovant and Neurocrine
Biosciences. $\mathrm{CH}$ has received speaking honoraria and consulting fees from Boston Scientific, Medtronic, and NeuroPace. PT works as consultant for Boston Scientific Neuromodulation and Gretap AG and is inventor on a number of patents for non-invasive neuromodulation. BM is employed by Engineering Acoustics who manufacture vibrotactile systems. No further disclosures.

The remaining authors declare that the research was conducted in the absence of any commercial or financial relationships that could be construed as a potential conflict of interest.

Copyright (c) 2021 Pfeifer, Kromer, Cook, Hornbeck, Lim, Mortimer, Fogarty, Han, Dhall, Halpern and Tass. This is an open-access article distributed under the terms of the Creative Commons Attribution License (CC BY). The use, distribution or reproduction in other forums is permitted, provided the original author(s) and the copyright owner(s) are credited and that the original publication in this journal is cited, in accordance with accepted academic practice. No use, distribution or reproduction is permitted which does not comply with these terms. 